\title{
Tabla de frecuencias y espectros de absorción infrarroja de compuestos relacionados con la química del cemento
}

\author{
T. MAZQLEZ-MORENO y M." T. BLANCO-VARULA \\ Dres. en Clentilas Quimitcas

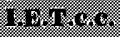

\section{INTRODUCCION}

En los núms. 175, 177 y 179 de esta Revista "Materiales de Construcción" se ha publicado un trabajo con el título "Aplicaciones prácticas de la espectroscopía de absorción infrarroja en el estudio de los crudos, del clínker y del cemento portland anhidro"; en él se decía textualmente que... "se dará, en forma de tablas las frecuencias y asignaciones correspondientes a los espectros IR tanto de los compuestos que se van estudiando como otros muchos que se consideran de interés. Asimismo se incluirán los respectivos espectros IR".

A causa de la extensión y de las propias características del anexo, se consideró oportuno el publicarlo separadamente, incluso bajo un título más explicativo.

\section{TABLA DE FRECUENCIAS}

En la Tabla que se adjunta se relacionan 66 compuestos que de una u otra forma tienen interés en la Química del Cemento. No es una relación exhaustiva, aunque pensamos que sí bastante completa.

Los valores de las frecuencias asignadas a los compuestos se obtienen a partir de:

- productos químicos R.A. (p. ejem. $\mathrm{CaSO}_{4} \cdot 2 \mathrm{H}_{2} \mathrm{O}$; gel de sílice);

- productos naturales $\left(\mathrm{SiO}_{2}\right.$-cuarzo $)$;

- productos sintetizados en este Laboratorio del IETcc.

En todos los casos se ha comprobado la pureza de los productos por técnicas distintas a $\mathrm{IR}$, como son difracción de rayos $\mathrm{X}$ y análisis térmicos.

- Estudios bibliográficos; en estos casos se dan las referencias oportunas.

En el esquema de frecuencias se aplican unas notaciones que pensamos dan una idea rápida de la intensidad y la forma de las bandas de absorción. Asimismo se indica con dos guiones (=) uniendo símbolos, en aquellos casos en los que las bandas están muy próximas y parcialmente solapadas.

Se podrían hacer numerosas observaciones de interés a la Tabla de frecuencias. Por ejemplo: 
- Hay compuestos hidratados con proporción variable de agua. La fórmula que se expresa

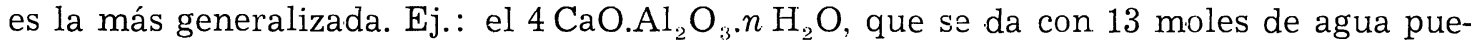
de aparecer al menos con 12 - 11 y 7 moles (11).

- Hay compuestos que se dan con una estequiometría definida y en realidad se pueden considerar como términos intermedios de disoluciones sólidas. Ej.: $6 \mathrm{CaO}_{2} \mathrm{Al}_{2} \mathrm{O}_{3}$. . $\mathrm{Fe}_{2} \mathrm{O}_{3}-4 \mathrm{CaO} \cdot \mathrm{Al}_{2} \mathrm{O}_{3} \cdot \mathrm{Fe}_{2} \mathrm{O}_{3}$ y el $6 \mathrm{CaO} \cdot \mathrm{Al}_{2} \mathrm{O}_{3} \cdot 2 \mathrm{Fe}_{2} \mathrm{O}_{3}$, que forman parte de la llamada "fase ferrítica del cemento portland".

- Según VOLANT (11) los aluminatos cálcicos con su mayor grado de hidratación presentan, en general, una amplia región de absorción bastante intensa, sin bandas claramente aisladas, entre 1.100 y $850 \mathrm{~cm}^{-1}$.

- Hay compuestos que siendo prácticamente puros, se identifican con determinadas fases del cemento. Ej.: $3 \mathrm{CaO} \mathrm{SiO}_{2}$, con "alita" y $2 \mathrm{CaO} . \mathrm{SiO}_{2}$, con belita. Se debe tener en cuenta que los espectros de esos compuestos difieren algo de sus respectivas fases.

Respecto a silicatos más o menos complejos del cemento es de interés el indicar que tienen como grupo fundamental el grupo $\mathrm{SiO}_{4}$, constituido por un tetraedro que tiene el $\mathrm{Si}$ en el centro del mismo y 4 átomos de oxígeno en los vértices. La asignación de las bandas IR de los espectros se realiza conforme a los modos de vibración del grupo de simetría $T_{\mathrm{a}}$. Por ello, de los cuatro modos normales de vibración, $\nu_{1}, \nu_{2}, \nu_{3}$ y $\nu_{4}$, son activos al IR $\nu_{3}$ y $\nu_{4}$, que son triplemente degenerados; $\nu_{2}$ es doblemente degenerado.

En los casos en los cuales la simetría de los tetraedros $\mathrm{SiO}_{4}$ es más baja que la teórica correspondiente a $T_{\mathfrak{a}}$, las bandas debidas a las vibraciones degeneradas podrían aparecer separadas. Es decir, no en forma de una banda observable en el espectro sino con 2 ó 3 bandas según sean doble o triplemente degeneradas.

Según esto, la aparición de varias bandas corrrespondientes a $\nu_{2}, \nu_{3}$, ó $\nu_{4}$, en el espectro de, p. ej.: el $\gamma-2 \mathrm{CaO} . \mathrm{SiO}_{2}$ indicará una cierta falta de simetría de la molécula.

Además la forma ancha de las bandas indica en general, un cierto desorden estructural, que en el caso de alitas y de belitas dan información sobre su potencial hidraulicidad.

- En el caso particular de la tobermorita $\left(\mathrm{CaO} \mathrm{SiO}_{2} \cdot n \mathrm{H}_{2} \mathrm{O}\right)$, como fase más importante del cemento hidratado, la banda más característica aparece generalmente ancha y entre valores que oscilan desde $920 \mathrm{~cm}^{-1}$ hasta casi $1.000 \mathrm{~cm}^{-1}$. Esta variación es función de un menor o mayor grado de polimerización de los tetraedros $\mathrm{SiO}_{4}$. Tanto la forma de la banda (más o menos ancha) como la frecuencia (más alta o más baja) a la que aparece, da una interesante información sobre las características "ligantes", de resistencia, que confiere al cemento portland endurecido.

Se adjuntan al presente trabajo 41 espectros de compuestos de importancia en la química de los cementos. No se dan todos los que se relacionan en la tabla de frecuencias al considerar que además de venir indicada la bibliografía correspondiente, se han publicado en los artículos de Materiales de Construcción a los que hacemos referencia en la Introducción del presente trabajo. 


\section{B I B L I O G R A F I A}

(1) Moenke, H.: Mineralspektren. Academie-verlag - Berlín (1966).

(2) GadsDen, J. A.: Infrared Spectra of Minerals and Related Inorganic Compounds - Butterworth Groups. London (1975).

(3) Kurczyk, H. G. y Schwiete, M. E.: Proceeding of the 4. ${ }^{\mathrm{a}}$ Int. Symp. Chem. of Cement, pág. 358. Washington (1960).

(4) Kalousek, G. L. y Roy, R.: Journal of the Amer. Ceram. Soc. vol. 40, n. 7, pág. 236 (1957).

(5) Bensted, J. y Varma, S. P.: World Cement Technology. vol. 8, n. 1, pág. 16 (1977).

(6) Bensted, J. y Varma, S. P.: Il Cemento, n.” 1 (1976).

(7) Bensted, J. y Varma, S. P.: Cement Technology, pág. 112, mayo-junio (1973).

(8) Bensted, J. y Varma, S. P.: Silicates Industriels, v. 38, n. 2 (1973).

(9) Bensted, J. y Varma, S. P.: Cement Technolgy, v. 5, n..$^{\circ}$, pág. 378 (1974).

(10) Nyquist, R. A. y KAGEL, R. O.: Infrared Spectra of Inorganic Compounds Academic Press. New York, London (1971).

(11) Frederickson, L. D.: Analytical Chemistry, v. 26, pág. 1883, diciembre (1954).

(12) Volant, J.: Tesis Doctoral. Facultad de Ciencias. Universidad de París (1966).

(13) Blanco-Varela, T. y Vazquez, T.: Materiales de Construción n.” 181 (1981).

(14) Blanco-Varela, T.: Tesis Doctoral. Universidad de Valladolid (1980).

(15) Vazquez, T.: Tesis Doctoral. Facultad de Ciencias. Universidad Complutense. Madrid (1975).

(16) VAzQuez, T.: Cuadernos de Investigación del IETcc, n.॰ 22 (1969).

(17) TARte, P.: Chimie Minerale. T. 1 p. 425 (1964). 


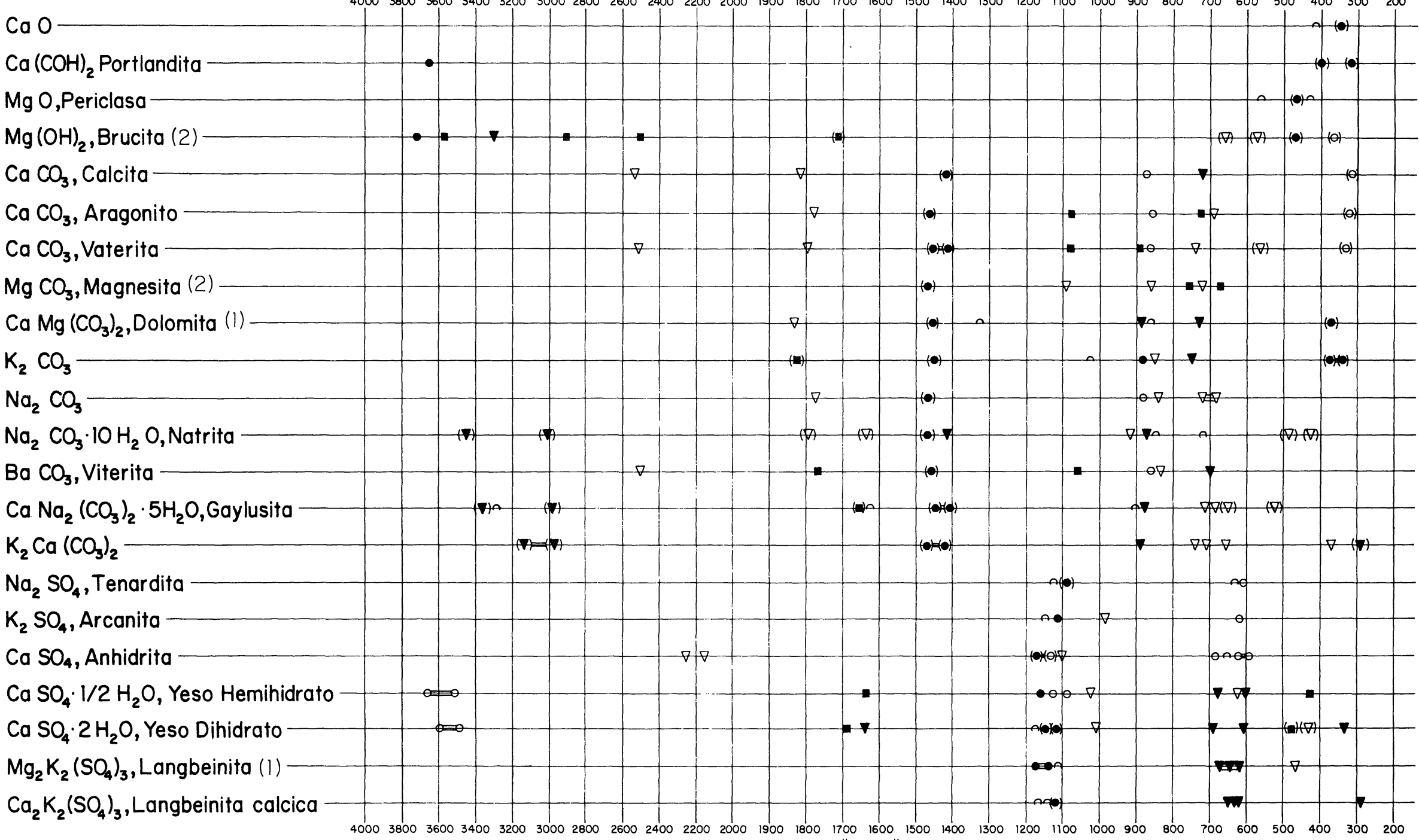
SIMBOLOS UTILIZADOS: Muy fuerte OFuerte $\mathbf{\nabla}$ Media DebIl $\nabla$ Muy debil $\cap$ "Hombro" ( ) Banda ancha $=$ Bandas solapadas mutuamente. 


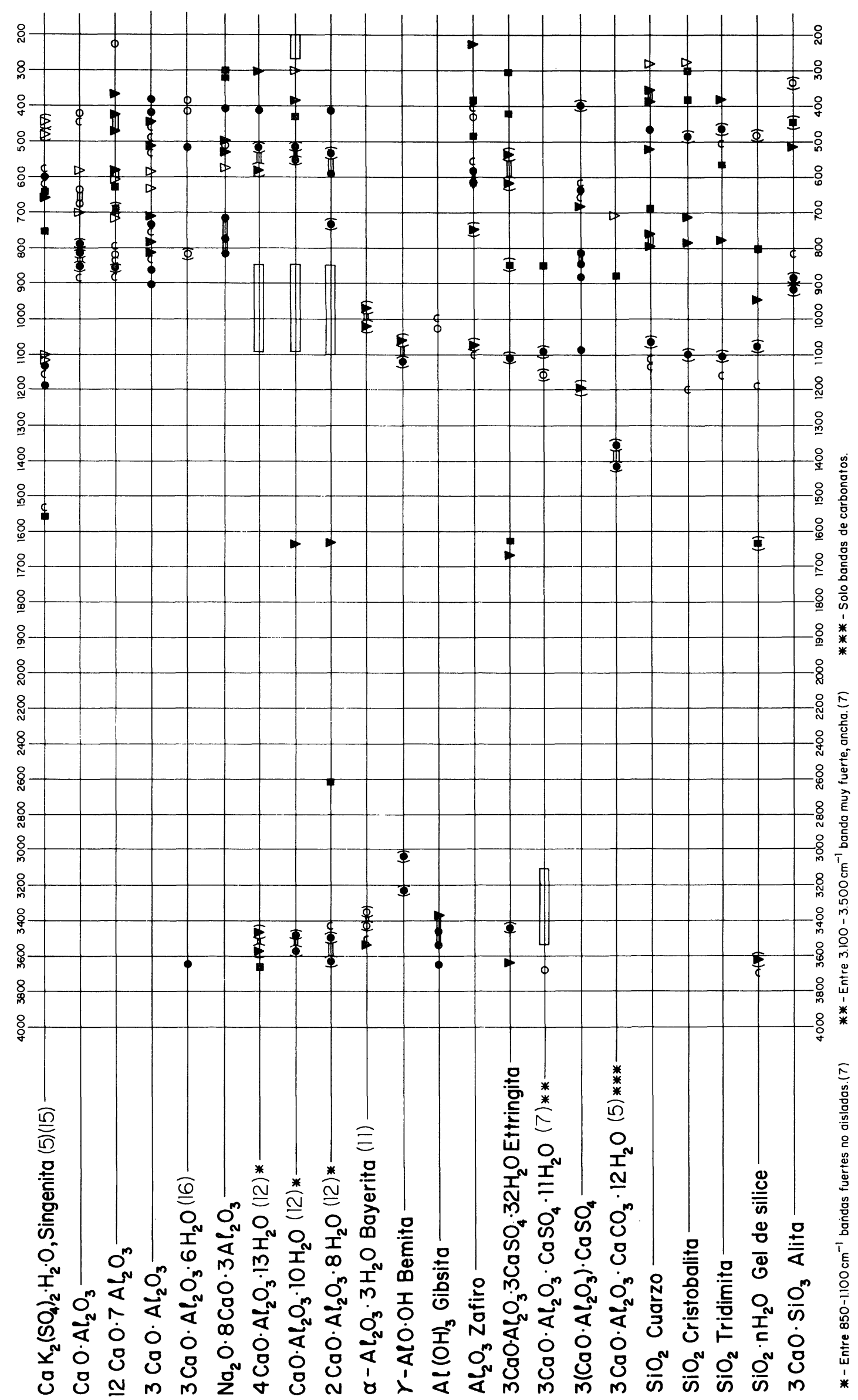

Materiales de Construccion N.॰ 182 - 1981 
$\mathrm{\beta} \cdot \mathrm{CaO} \cdot \mathrm{SiO}_{2}$ Wollastonita (14)

$\alpha \cdot \mathrm{CaO} \cdot \mathrm{SiO}_{2}$ Seudowollastonita $(14)$

$3 \mathrm{CaO} \cdot 2 \mathrm{SiO}_{2}$ Rankinita (14)

$\mathrm{CaO} \cdot \mathrm{SiO}_{2} \cdot \mathrm{nH}_{2} \mathrm{O}$ Tobermorita (3)(4)

$6\left[\mathrm{CaO} \cdot \mathrm{SiO}_{2}\right] \mathrm{H}_{2} \mathrm{O}$ Xonotlita (I)(4)

$3 \mathrm{CaO} \cdot 2 \mathrm{SiO}_{2} \cdot \mathrm{CaF}_{2}$ Cuspidina (14)

$\mathrm{Fe}_{2} \mathrm{O}_{3}(16)(17)$

$2 \mathrm{CaO} \cdot \mathrm{Fe}_{2} \mathrm{O}_{3}(16)(17)$

$6 \mathrm{CaO} \cdot 2 \mathrm{Al}_{2} \mathrm{O}_{3} \cdot \mathrm{Fe}_{2} \mathrm{O}_{3}(17)$

$4 \mathrm{CaO} \cdot \mathrm{Al}_{2} \mathrm{O}_{3} \cdot \mathrm{Fe}_{2} \mathrm{O}_{3}(15)(17)$

$6 \mathrm{CaO} \cdot \mathrm{Al}_{2} \mathrm{O}_{3} \cdot 2 \mathrm{Fe}_{2} \mathrm{O}_{3}(15)(17)$

$2\left(2 \mathrm{CaO} \cdot \mathrm{SiO}_{2}\right) \cdot \mathrm{CaF}_{2}(13)$

$2\left(2 \mathrm{CaO} \cdot \mathrm{SiO}_{2}\right) \cdot \mathrm{CaSO}_{4}(13)$

$2\left(2 \mathrm{CaO} \cdot \mathrm{SiO}_{2}\right) \cdot \mathrm{CaCO}_{3}$ Spurrita (5)

$3 \mathrm{CaO} \cdot \mathrm{Al}_{2} \mathrm{O}_{3} \cdot \mathrm{CaCl}_{2} \cdot \mathrm{OOH}_{2} \mathrm{O}$ Sal de Friedel $3 \mathrm{Ca}_{2} \mathrm{SiO}_{4} \cdot 3 \mathrm{CaSO}_{4} \cdot \mathrm{CaF}_{2}$ Fluor ellestadita $(13)$ $\mathrm{CaSiO}_{3} \cdot \mathrm{CaCO}_{3} \cdot \mathrm{CaSO}_{4} \cdot 15 \mathrm{H}_{2} \mathrm{O}$ Taumasita (I)(8)

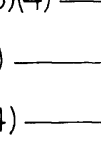

\begin{tabular}{llllll|l|l|l|l|l|l|l|l|l|l|l|} 
& & & & & & & & & & & & & & \\
\hline
\end{tabular}
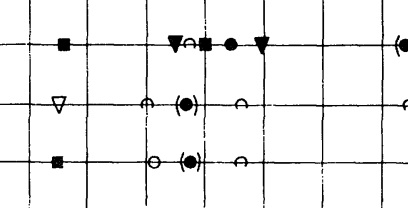


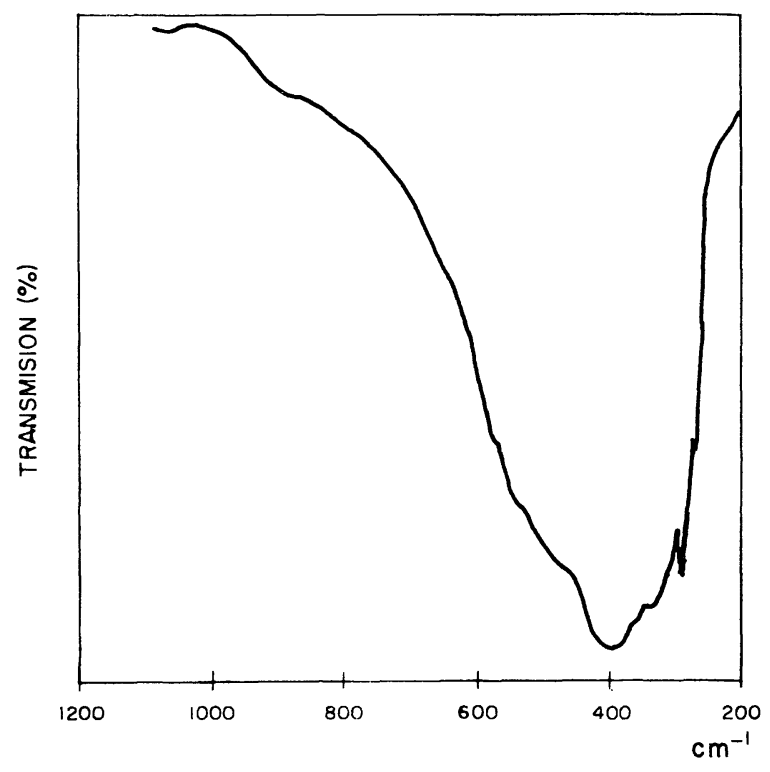

Fig. 1. $-\mathrm{CaO}$.

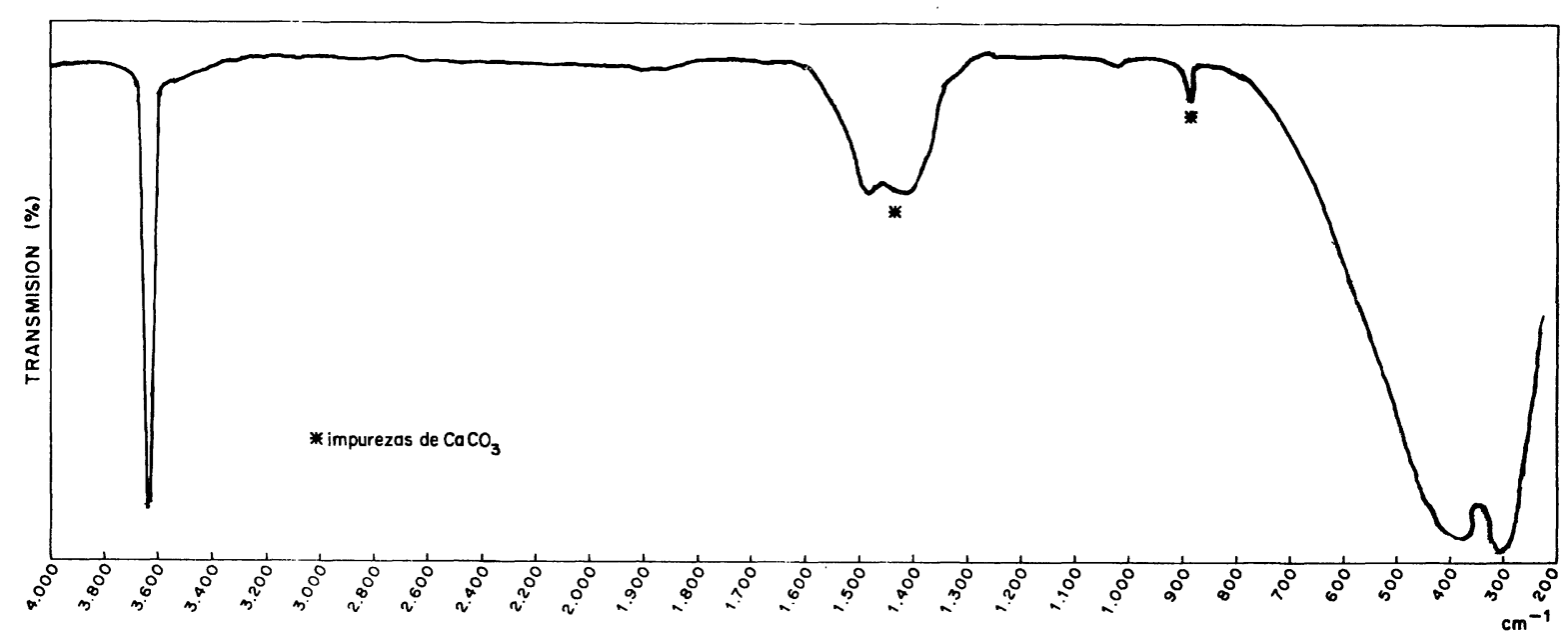

Fig. 2.- $\mathrm{Ca}(\mathrm{OH})_{2}$; potlandita (con impurezas de $\mathrm{CaCO}_{3}$ ).

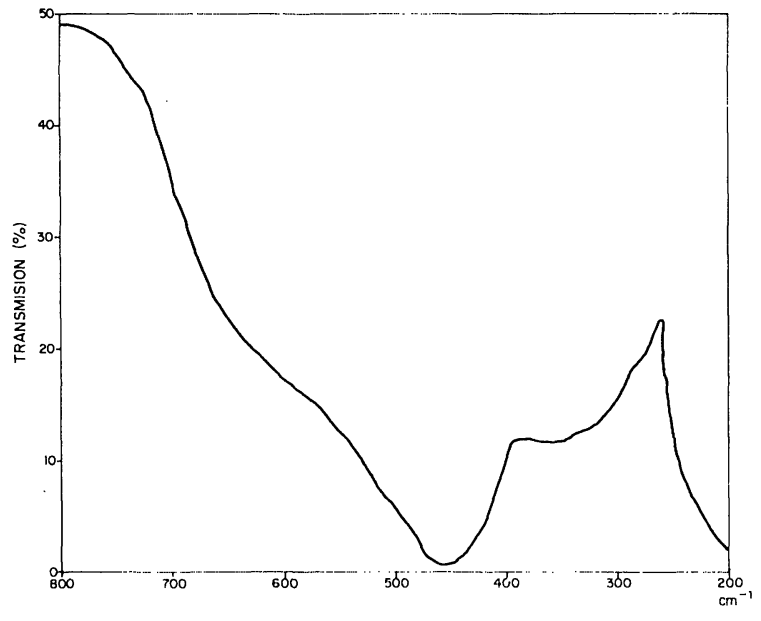

Fig. 3.-MgO ; periclasa.

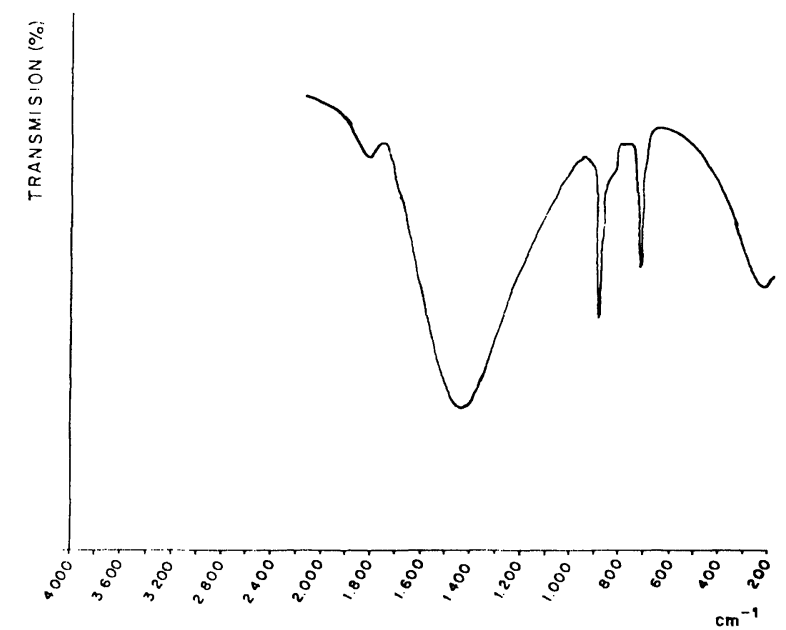

Fig. 4. $-\mathrm{CaMg}\left(\mathrm{CO}_{3}\right)_{2}$; dolomita (1). 


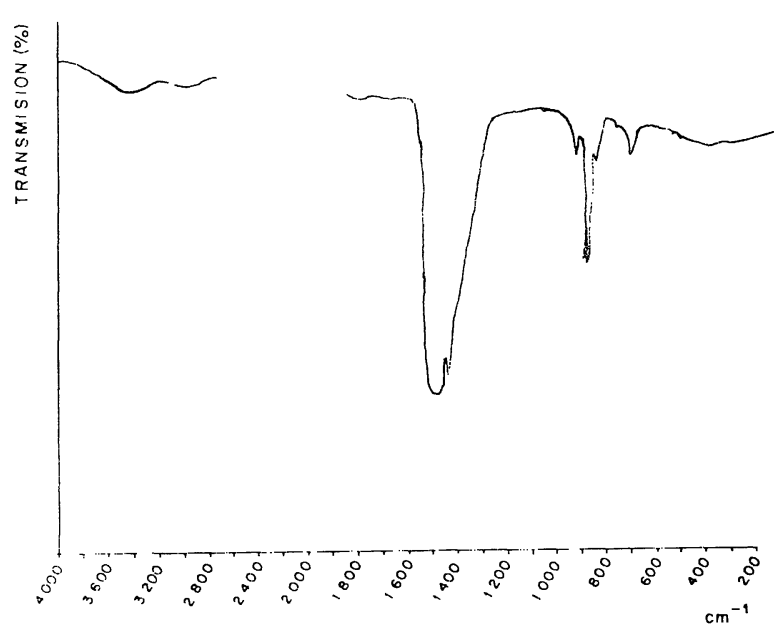

Fig. 5.- $-\mathrm{Na}_{2} \mathrm{CO}_{3} .10 \mathrm{H}_{2} \mathrm{O}$; natrita (1).

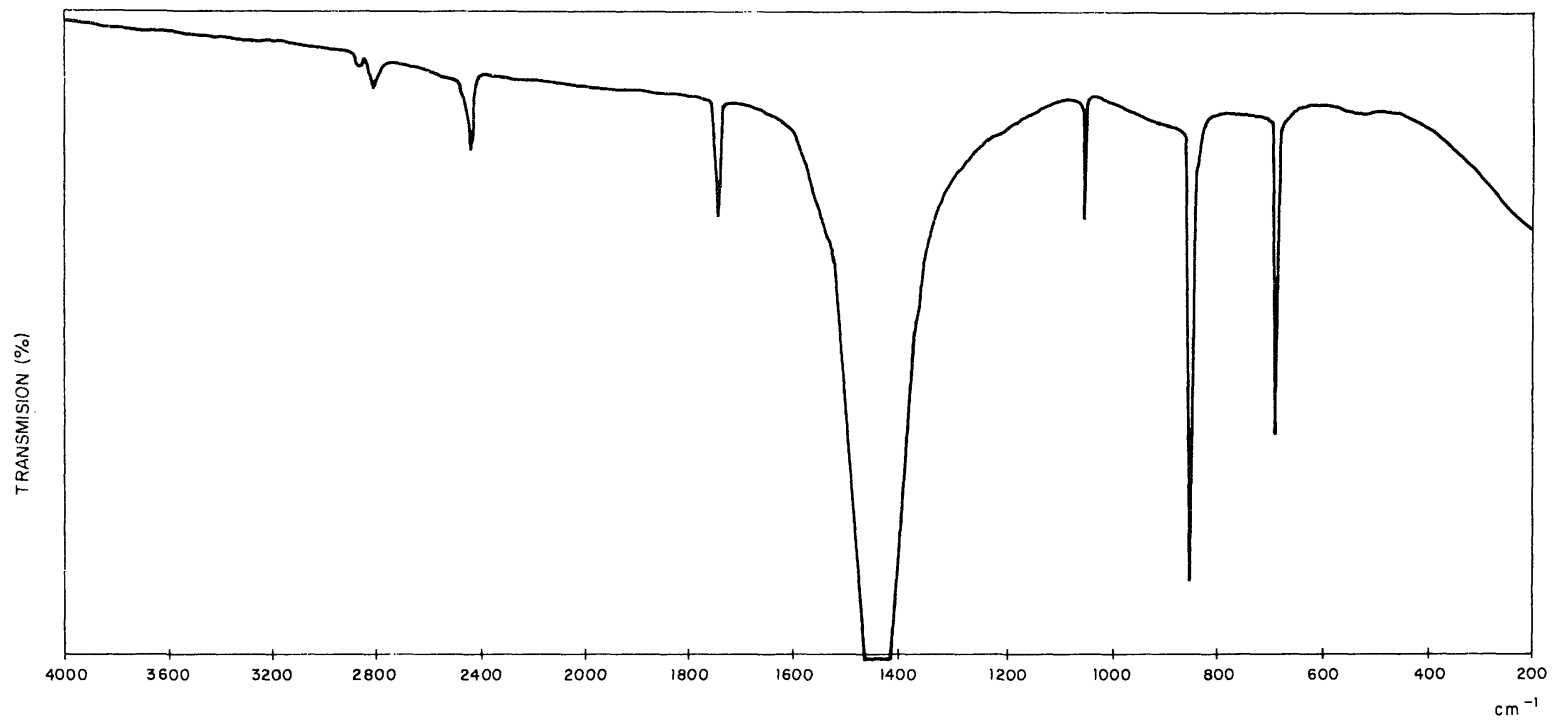

Fig. 6. $-\mathrm{BaCO}_{3}$; viterita.

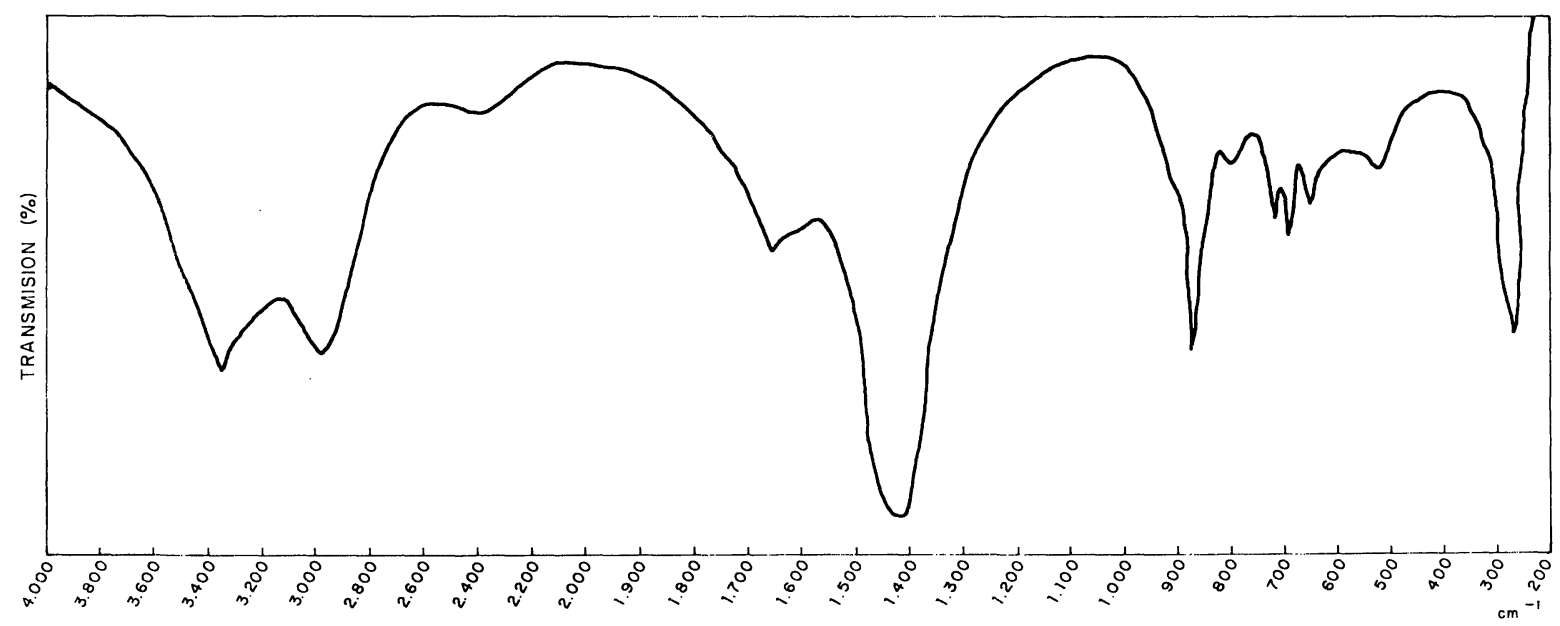

Fig. 7.- $\mathrm{CaNa}_{2}\left(\mathrm{CO}_{3}\right)_{2} .5 \mathrm{H}_{2} \mathrm{O}$; Gaylusita. 


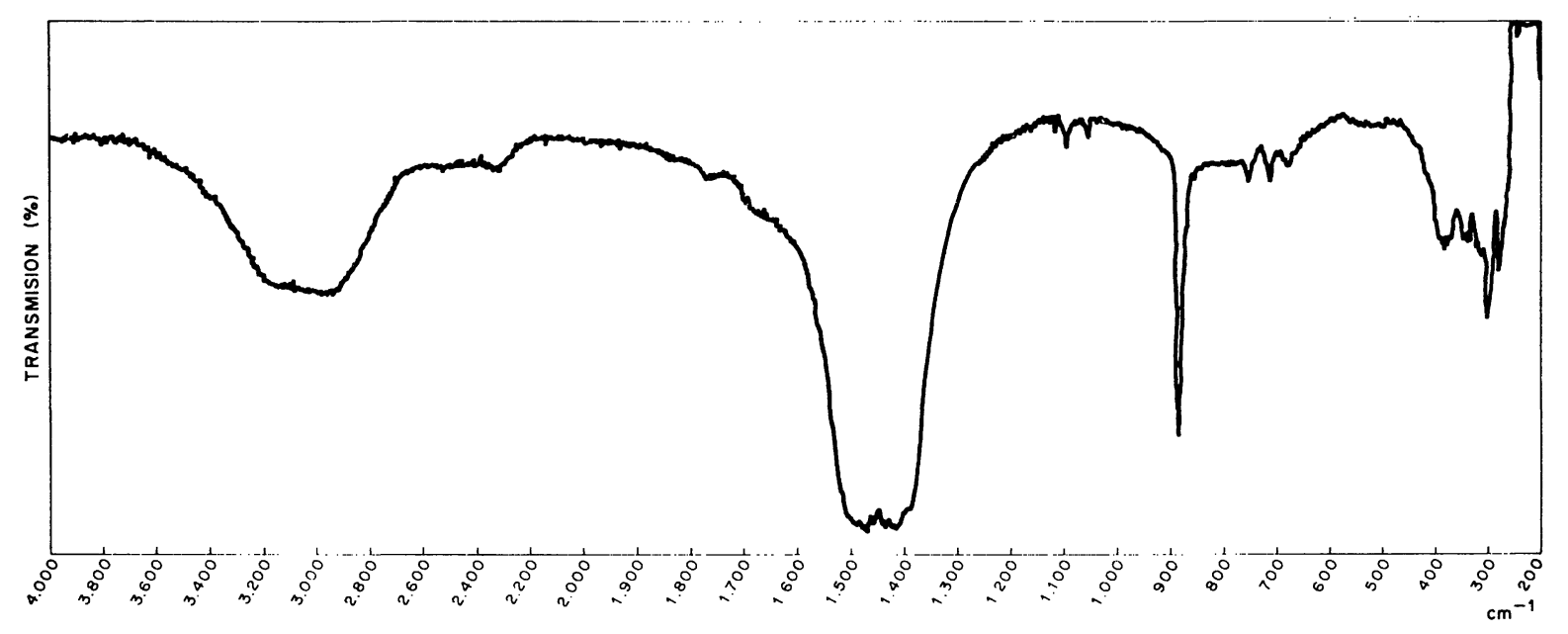

Fig. 8. $-\mathrm{K}_{2} \mathrm{Ca}\left(\mathrm{CO}_{3}\right)_{2} . n \mathrm{H}_{2} \mathrm{O}$.

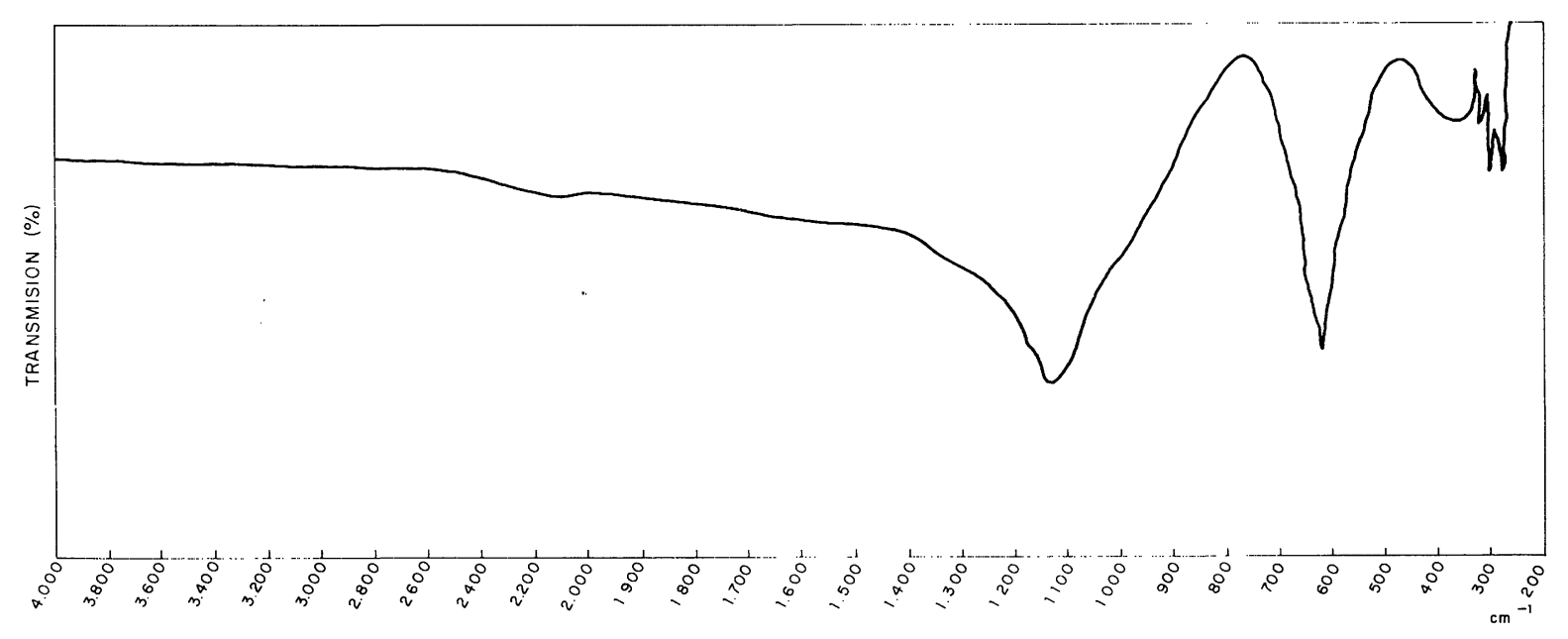

Fig. 9.- $\mathrm{Na}_{2} \mathrm{SO}_{4}$; tenardita.

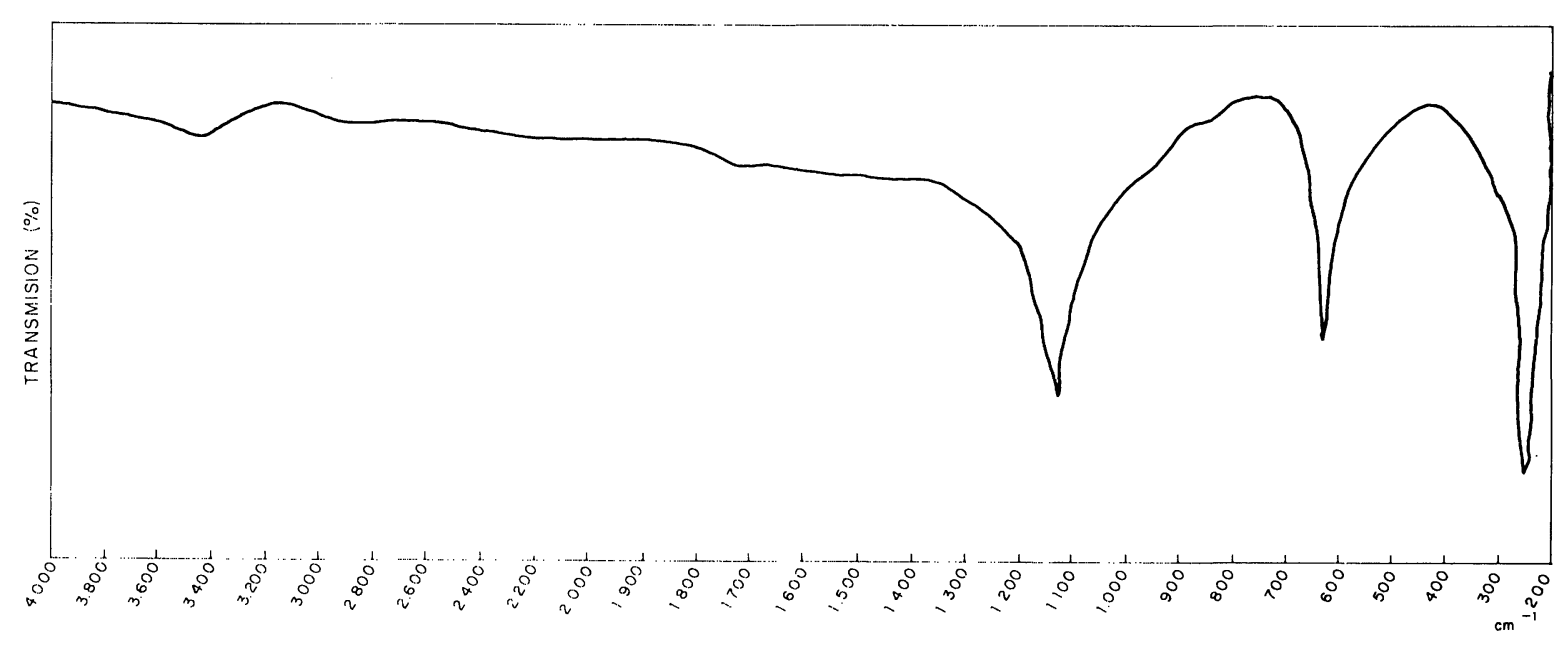

Fig. 10. $-\mathrm{K}_{2} \mathrm{SO}_{4}$; arcanita. 


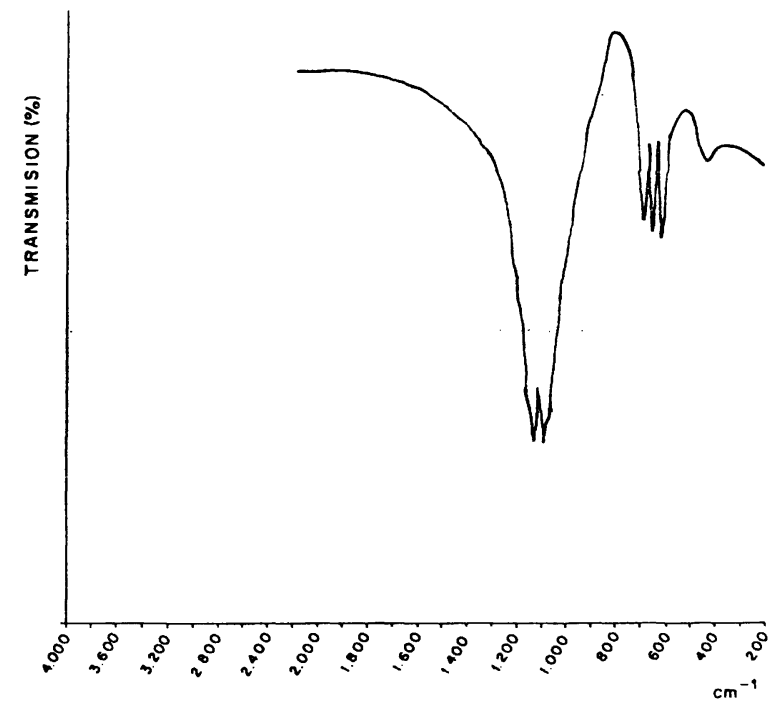

Fig. 11. $-\mathrm{Mg}_{2} \mathrm{~K}_{2}\left(\mathrm{SO}_{4}\right)_{3}$; langbeinita (1).

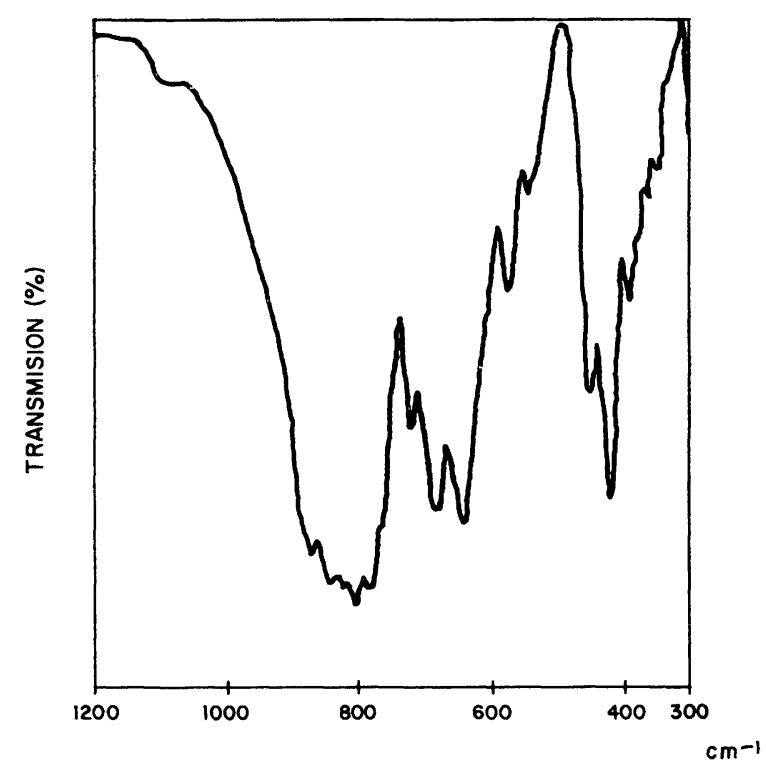

Fig. 13.- $\mathrm{CaO} \cdot \mathrm{Al}_{2} \mathrm{O}_{3}$.

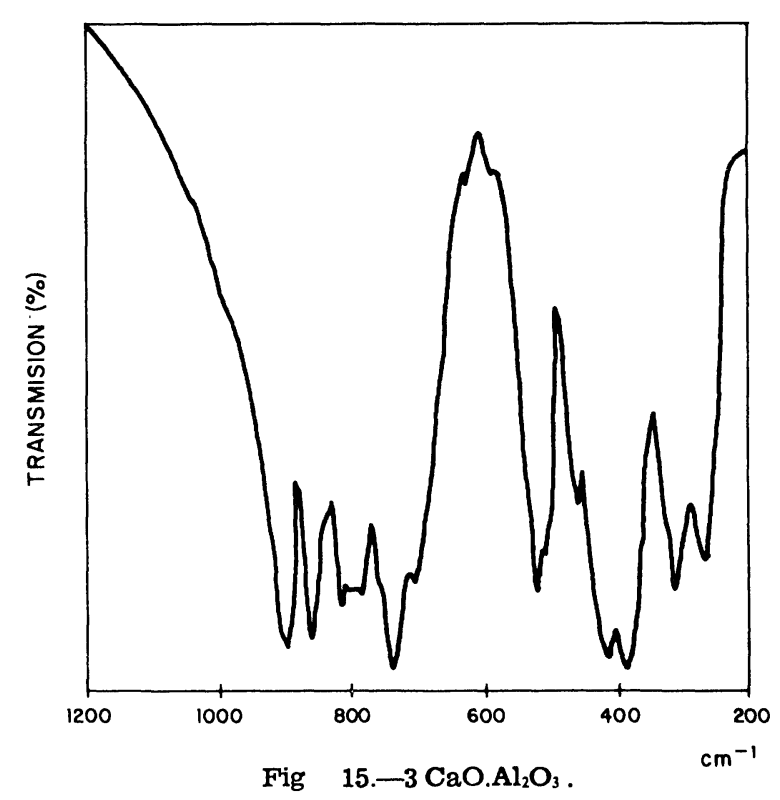

40

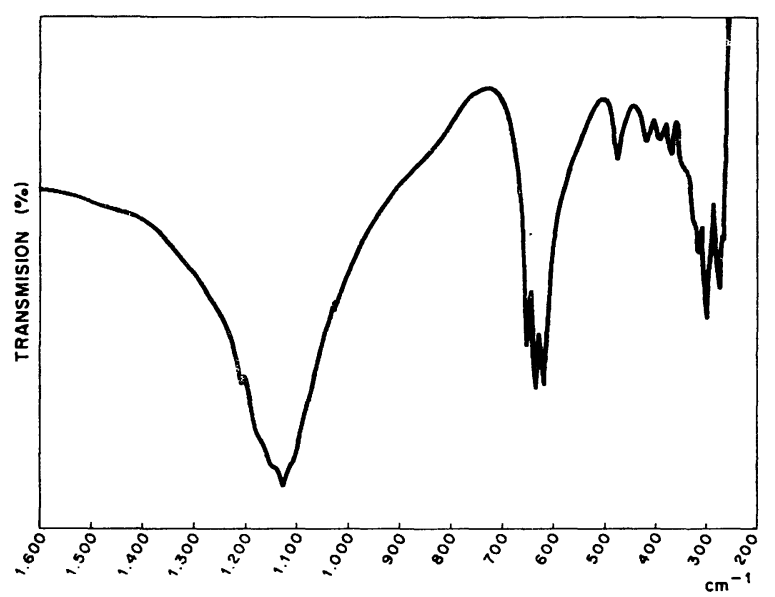

Fig. 12.- $\mathrm{Ca}_{2} \mathrm{~K}_{2}\left(\mathrm{SO}_{4}\right)_{3}$; langbeinita cálcica.

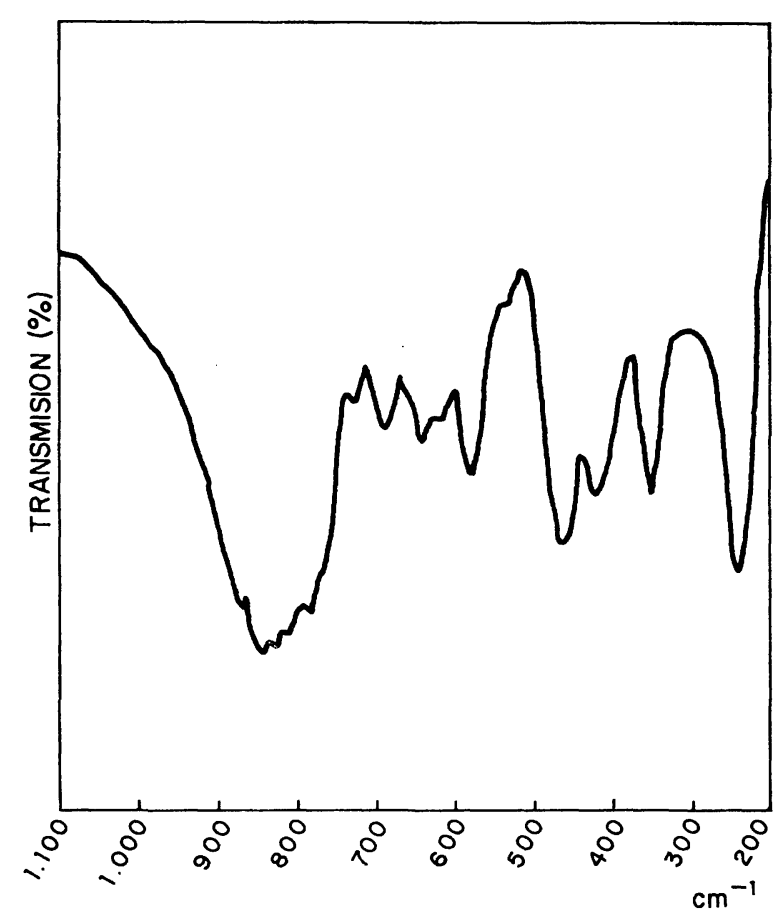

Fig. 14.-12 $\mathrm{CaO} .7 \mathrm{Al}_{2} \mathrm{O}_{3}$. 

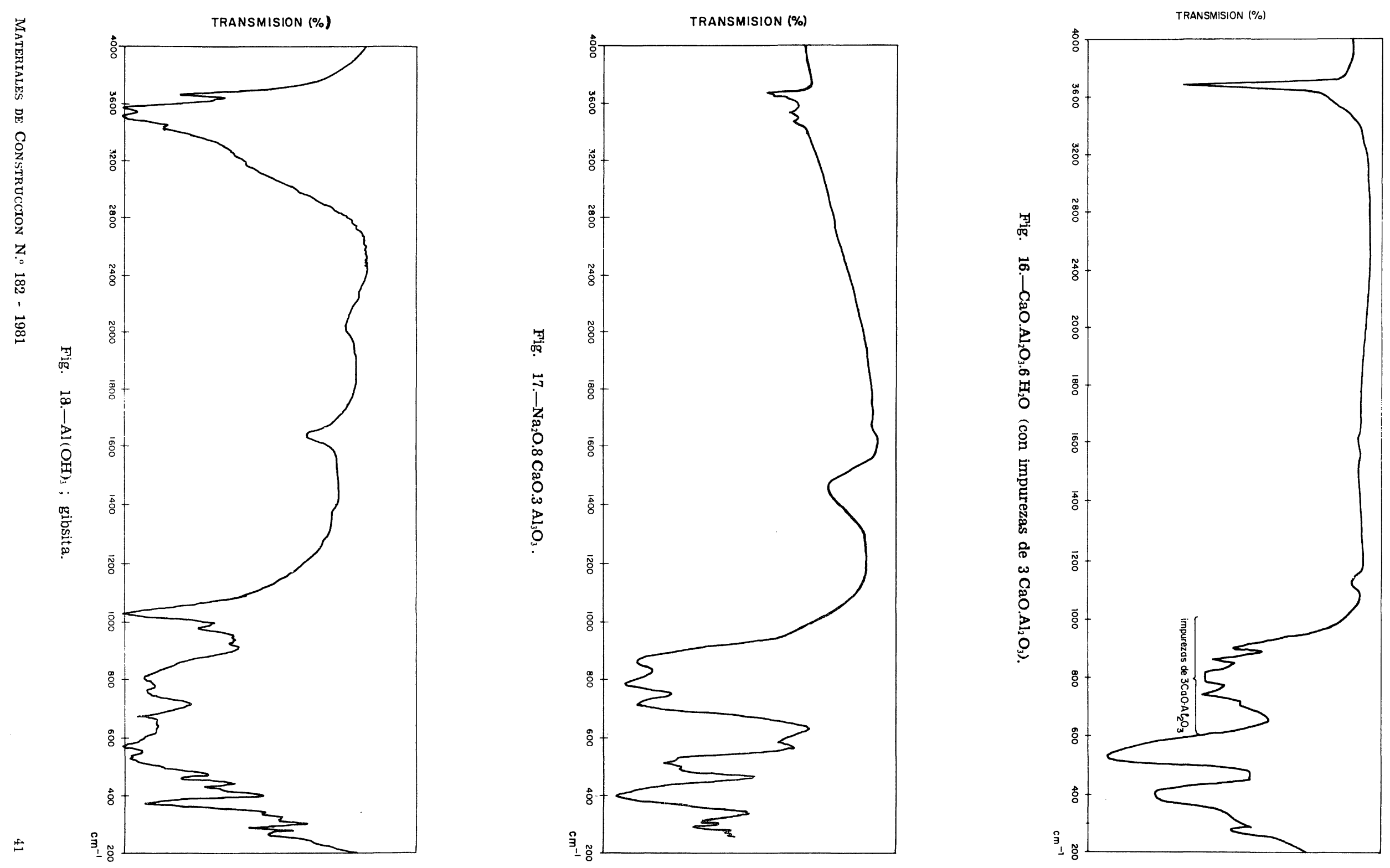


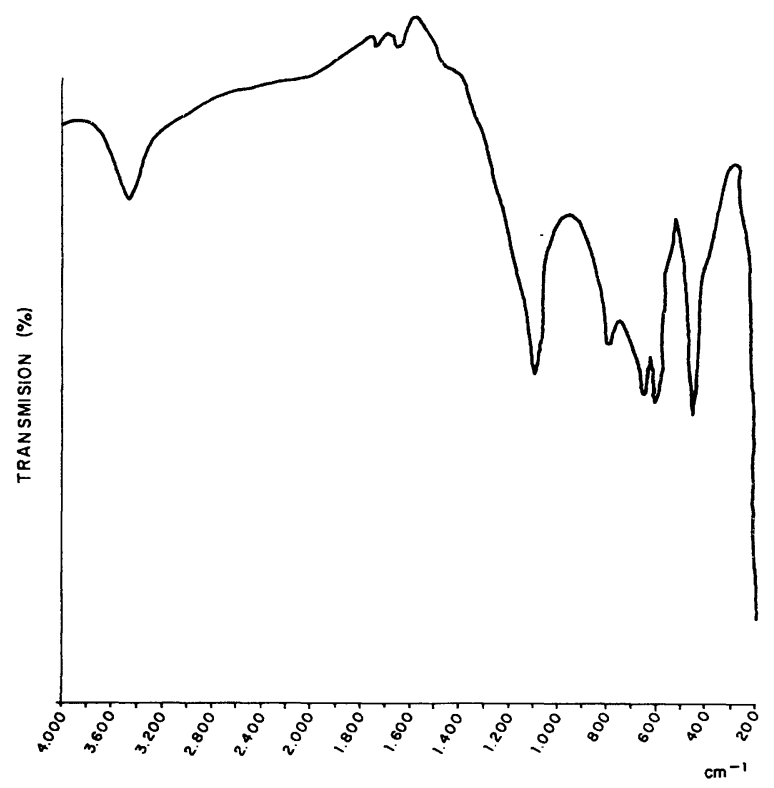

Fig. 19. $-\mathrm{Al}_{2} \mathrm{O}_{3}$.

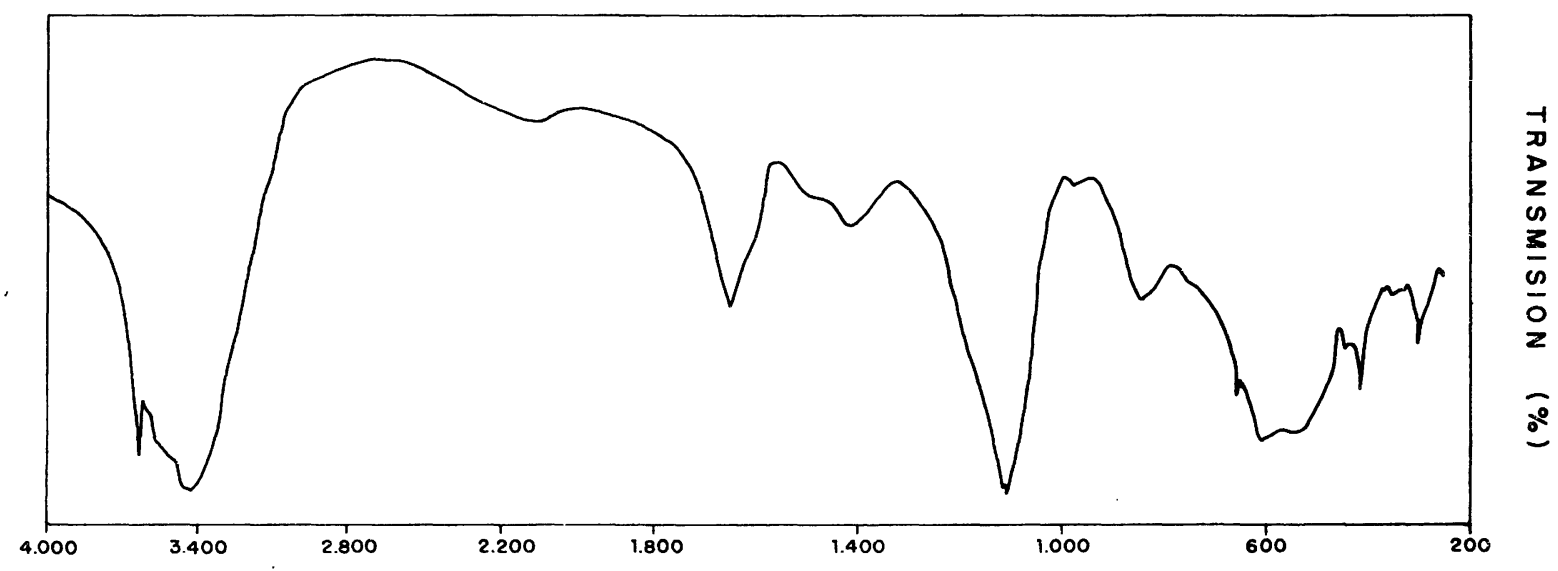

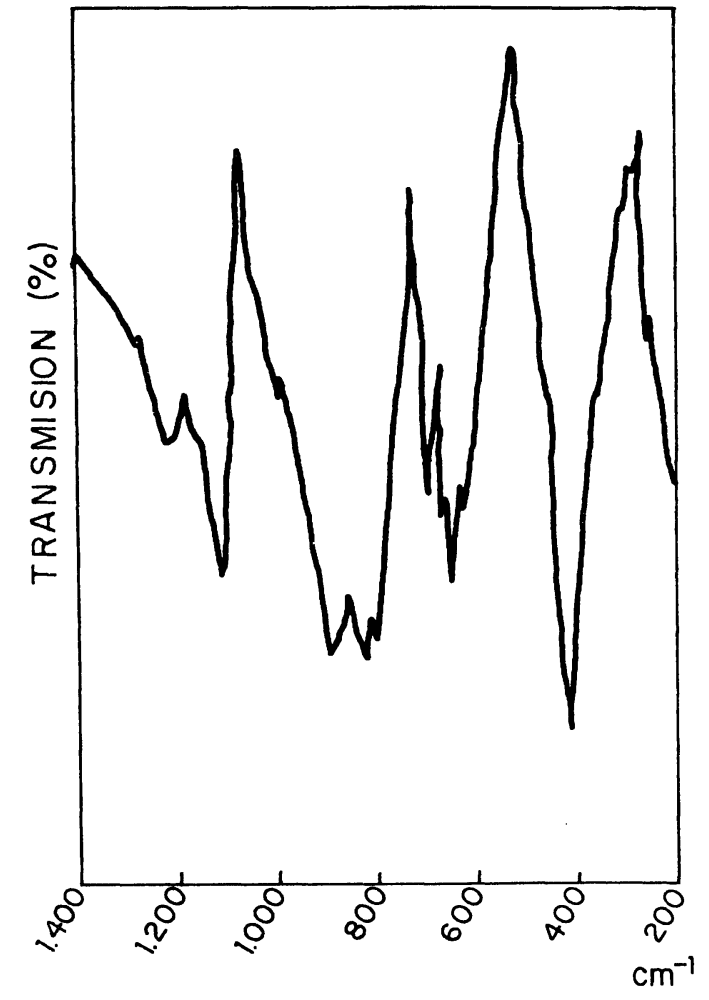

42

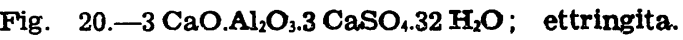

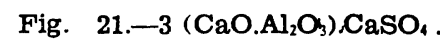




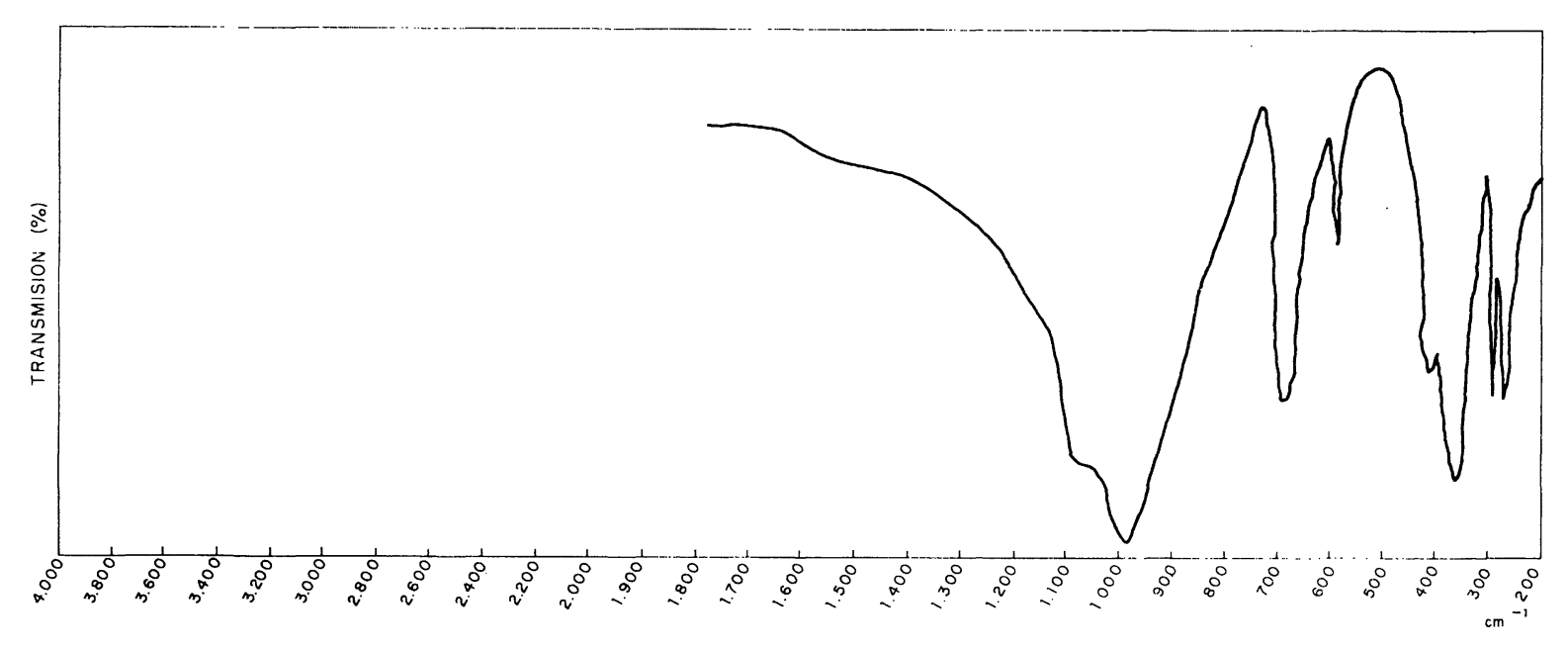

Fig. 22. $-\mathrm{SiO}_{2}$; cuarzo.

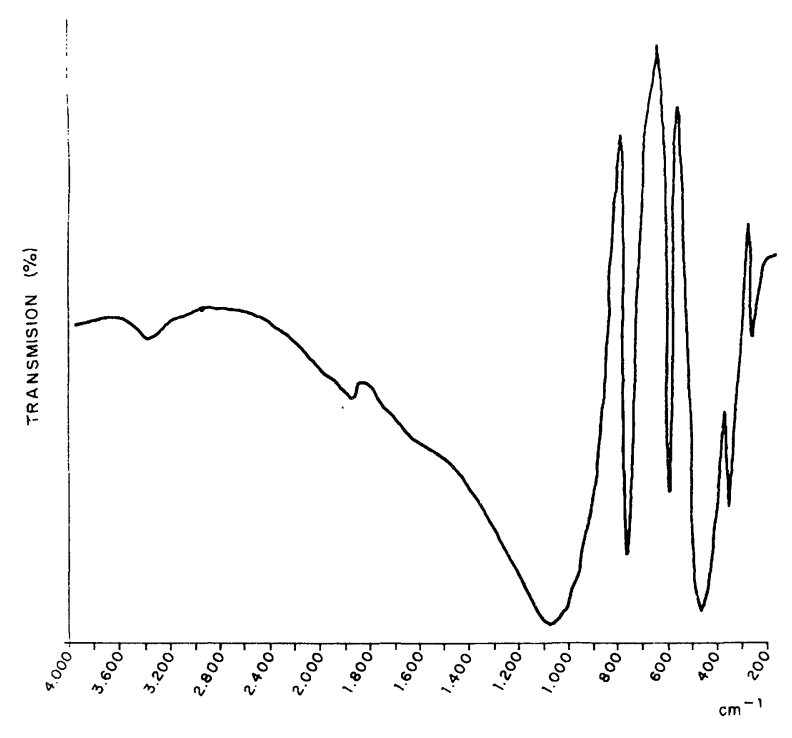

Fig. 23. $-\mathrm{SiO}_{2} ;$ cristobalita.

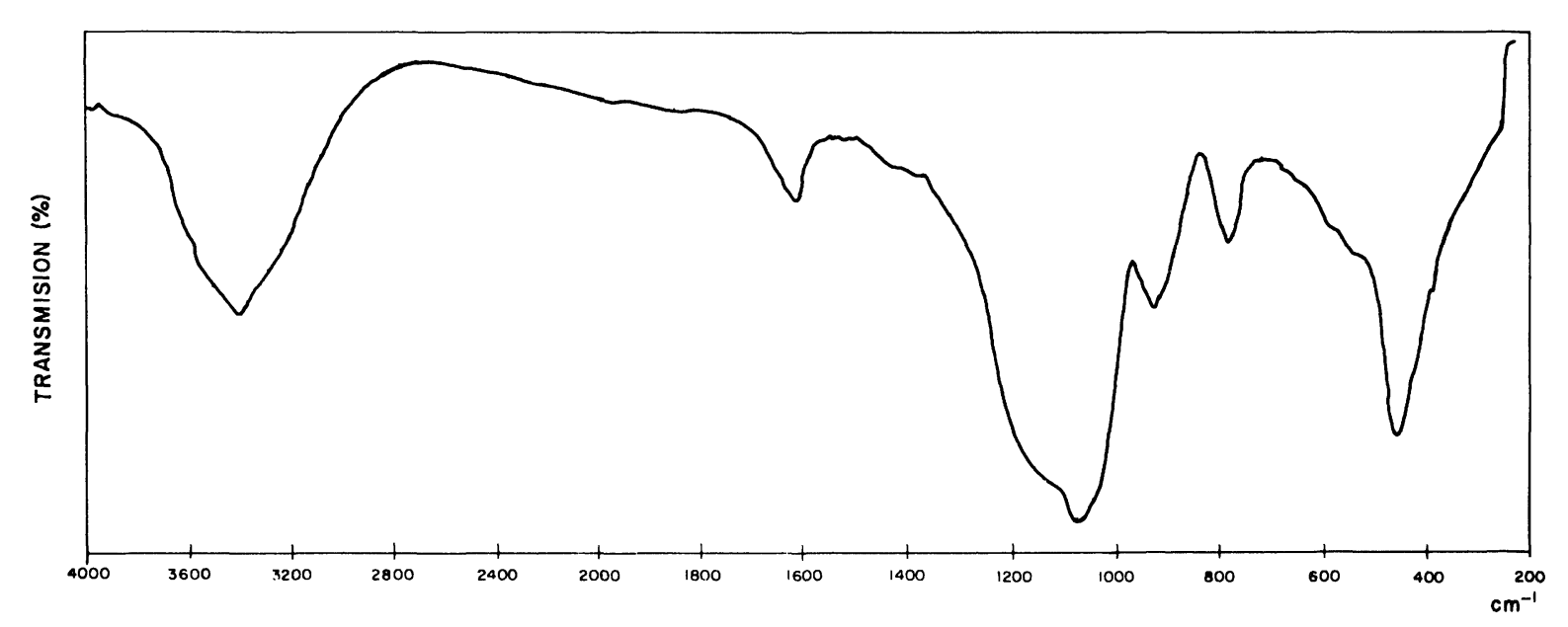

Fig. 24.- $-\mathrm{SiO}_{2} . n \mathrm{H}_{2} \mathrm{O}$; gel de sílice. 


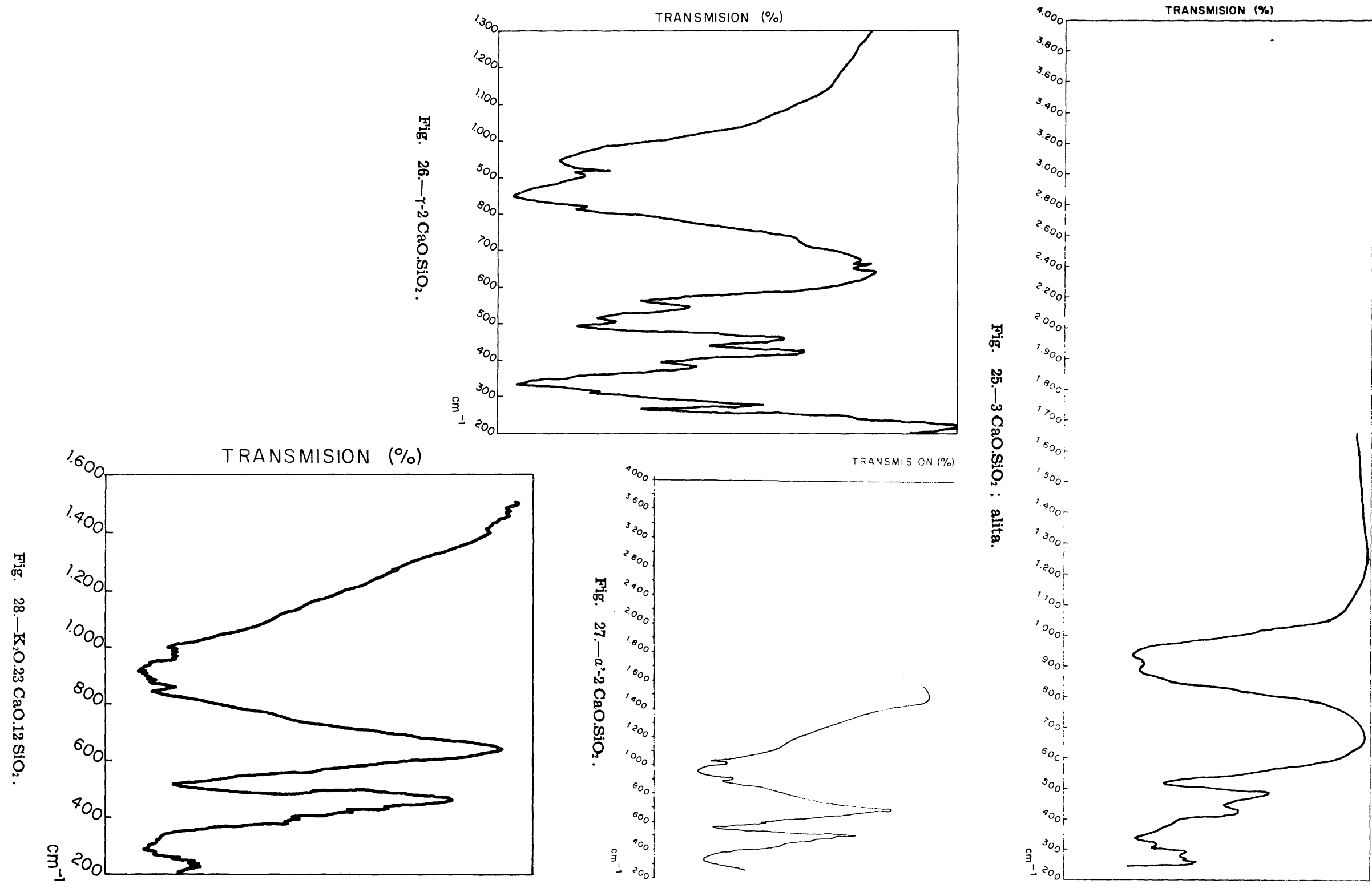




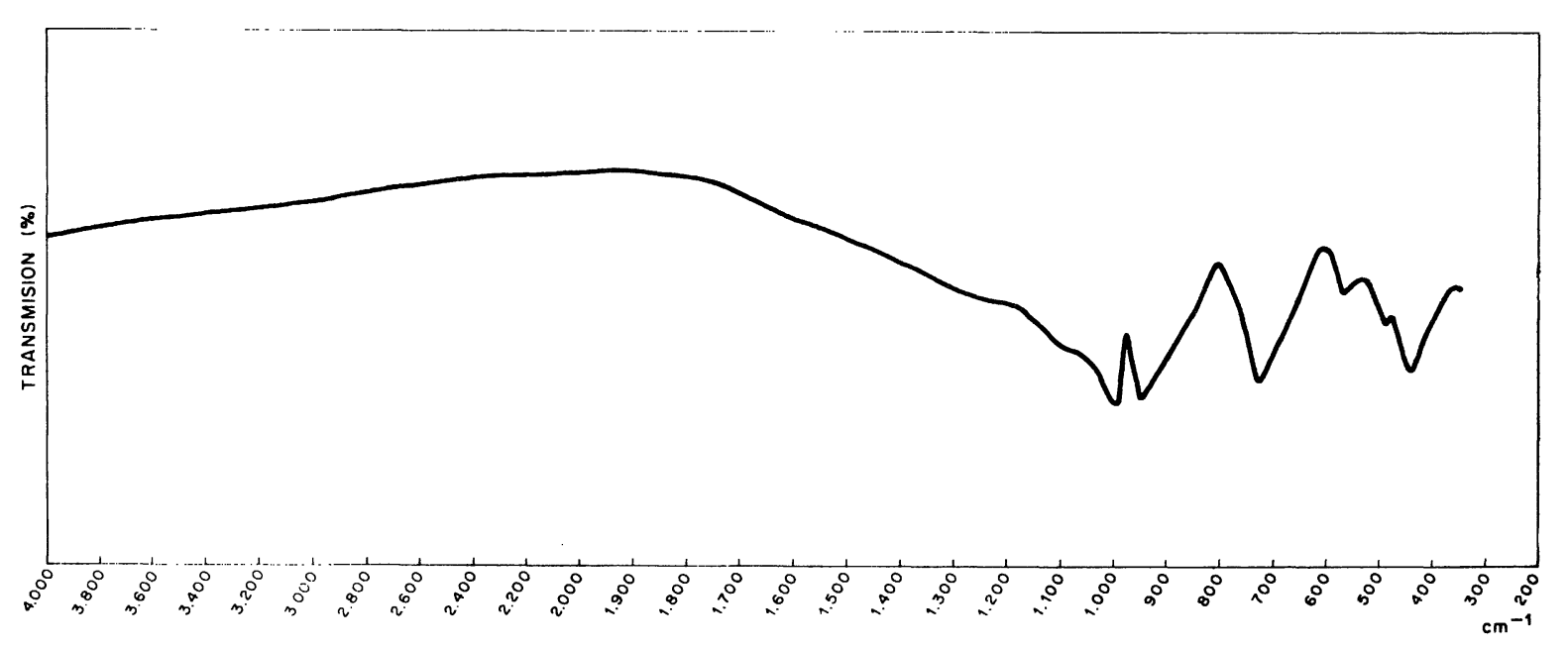

Fig. 29.- $\beta-\mathrm{CaO} . \mathrm{SiO}_{2}$; wollastonita.

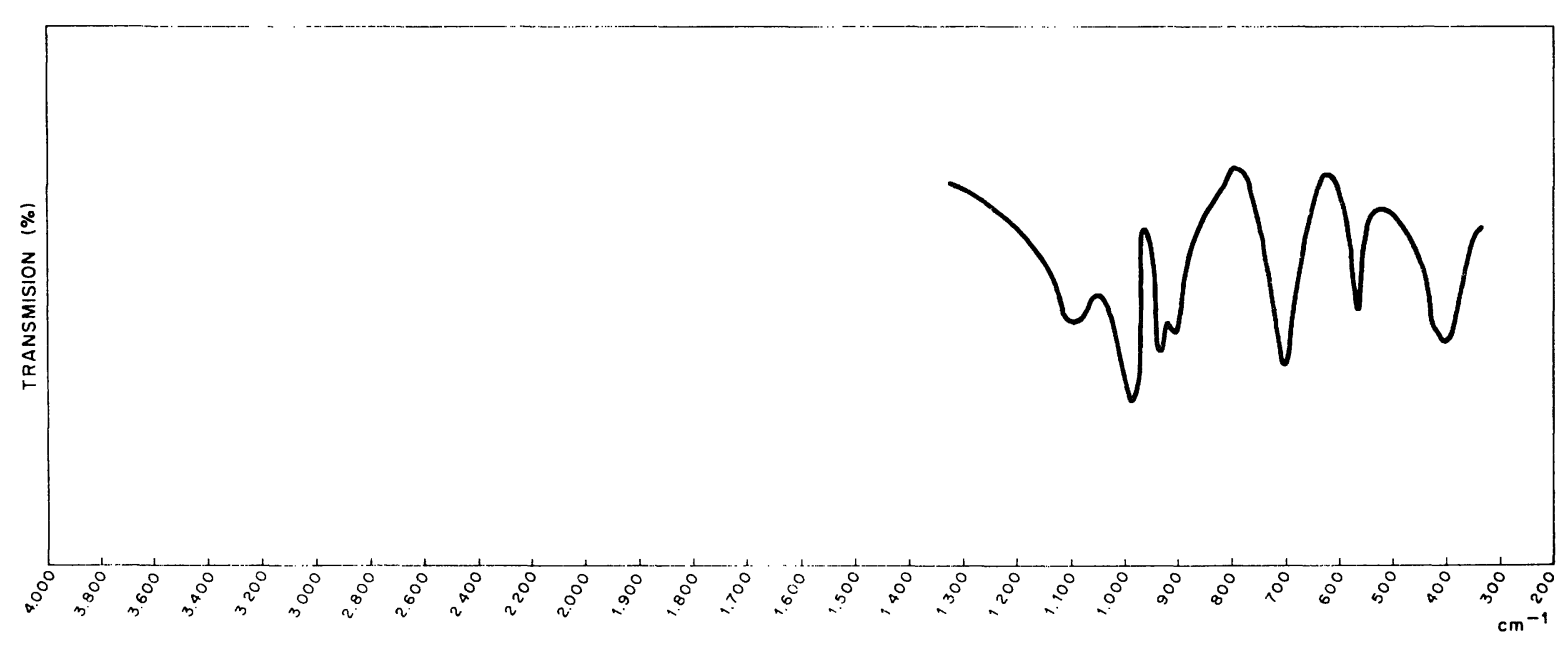

Fig. 30.- $\alpha-\mathrm{CaO} . \mathrm{SiO}_{2}$; seudowollastonita.

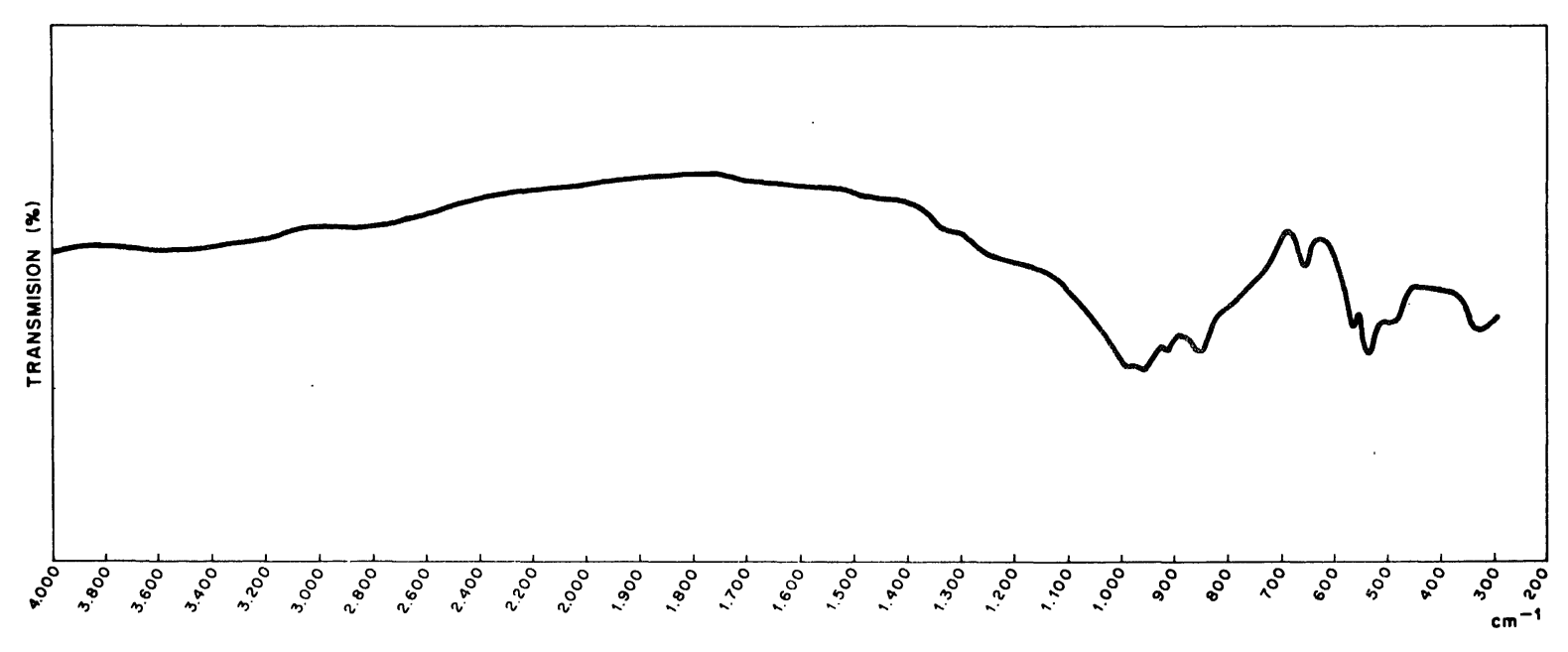

Fig. 31.-3 CaO.2 $\mathrm{SiO}_{2}$; rankinita. 


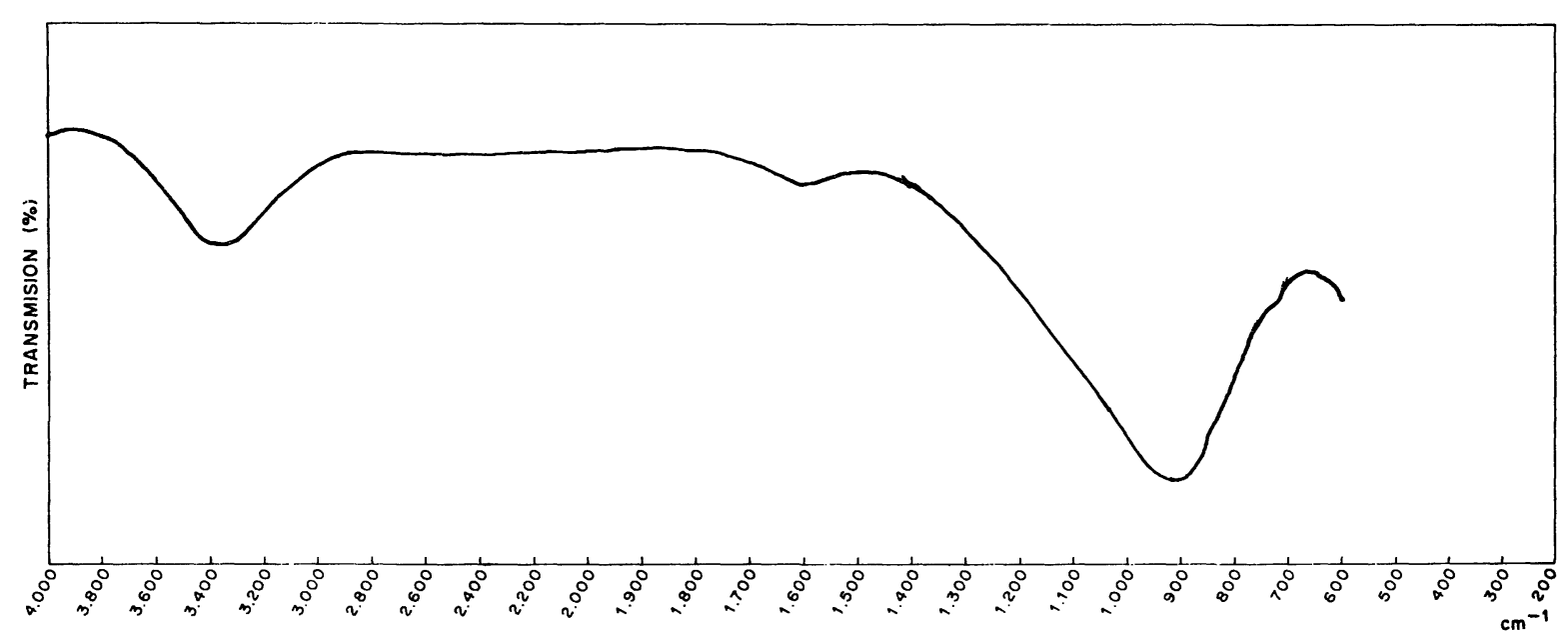

Fig. 32.- $\mathrm{CaO} . \mathrm{SiO}_{2} . n \mathrm{H}_{2} \mathrm{O}$; tohermorita (4).

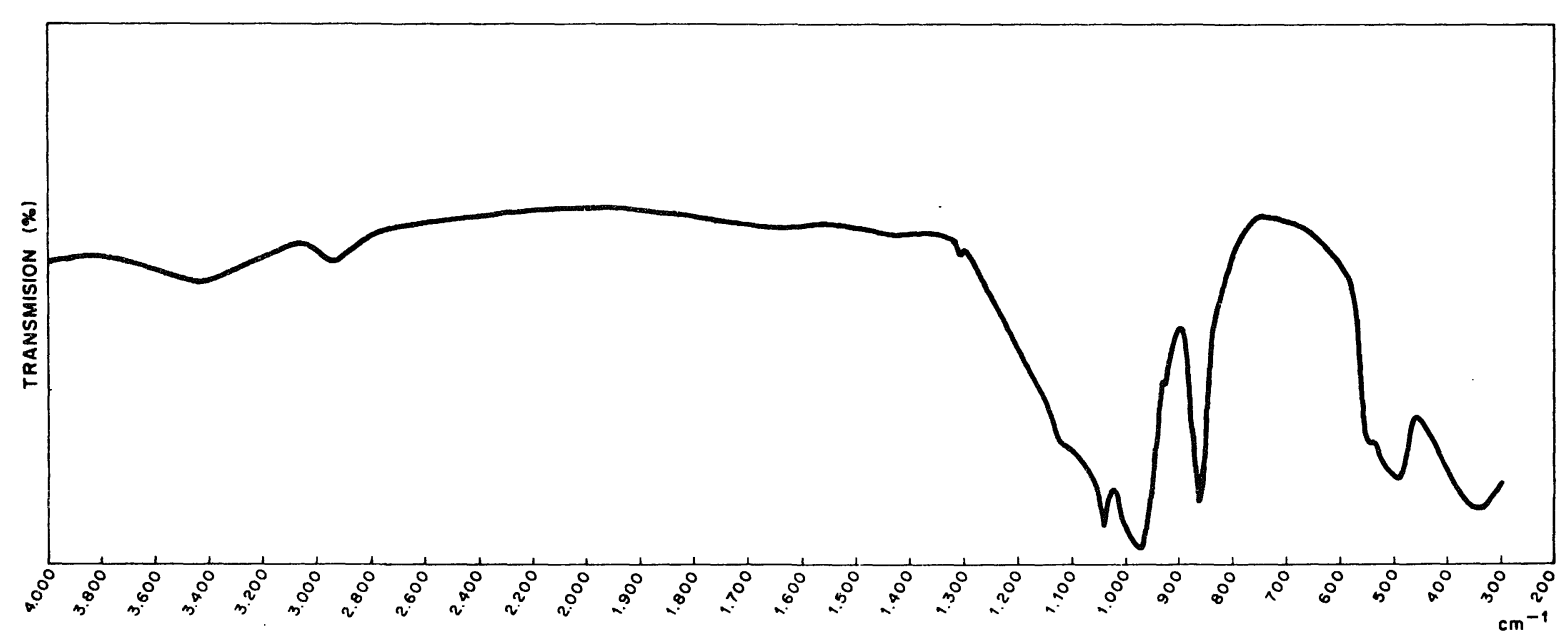

Fig. 33. $-3 \mathrm{CaO} .2 \mathrm{SiO}_{2} \cdot \mathrm{CaF}_{2}$; cuspidina.

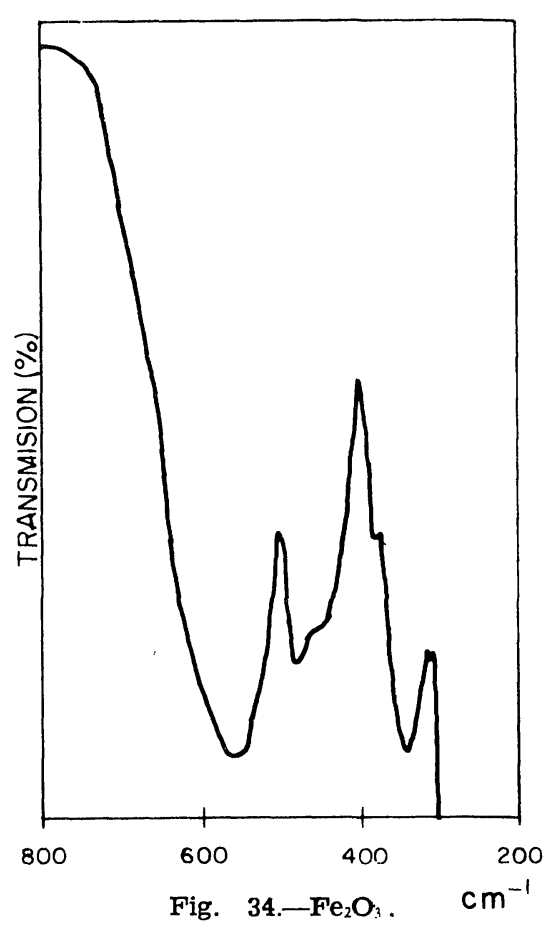

46

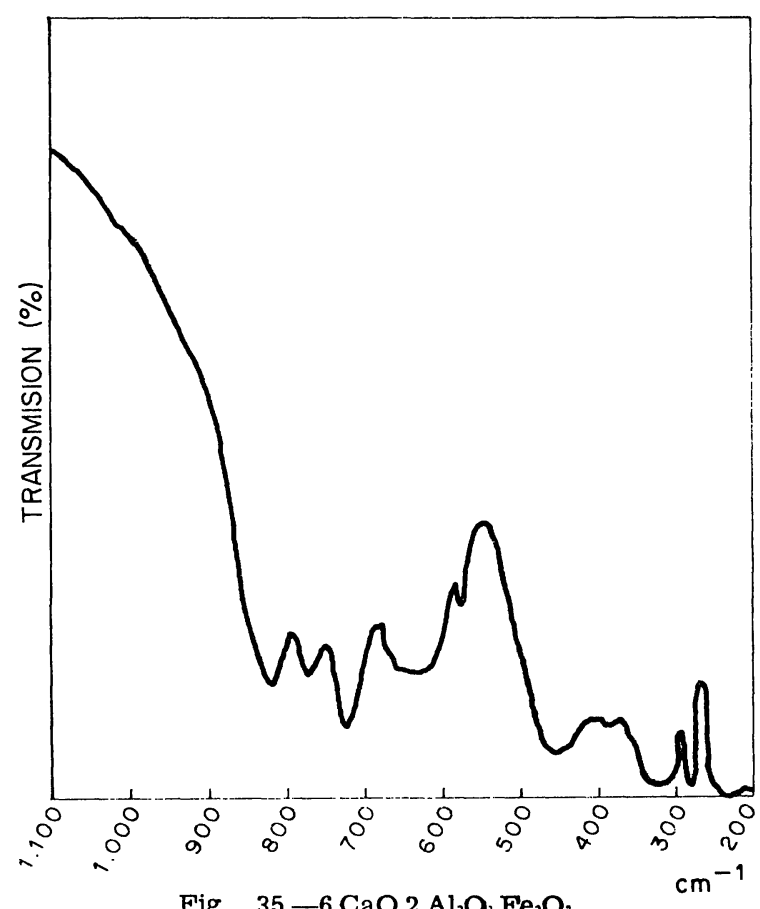

Fig. 35.-6 $\mathrm{CaO} .2 \mathrm{Al}_{2} \mathrm{O}_{3} \cdot \mathrm{Fe}_{2} \mathrm{O}_{3}$.

Materiales de Construccion N. ${ }^{\circ} 182-1981$ 


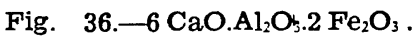
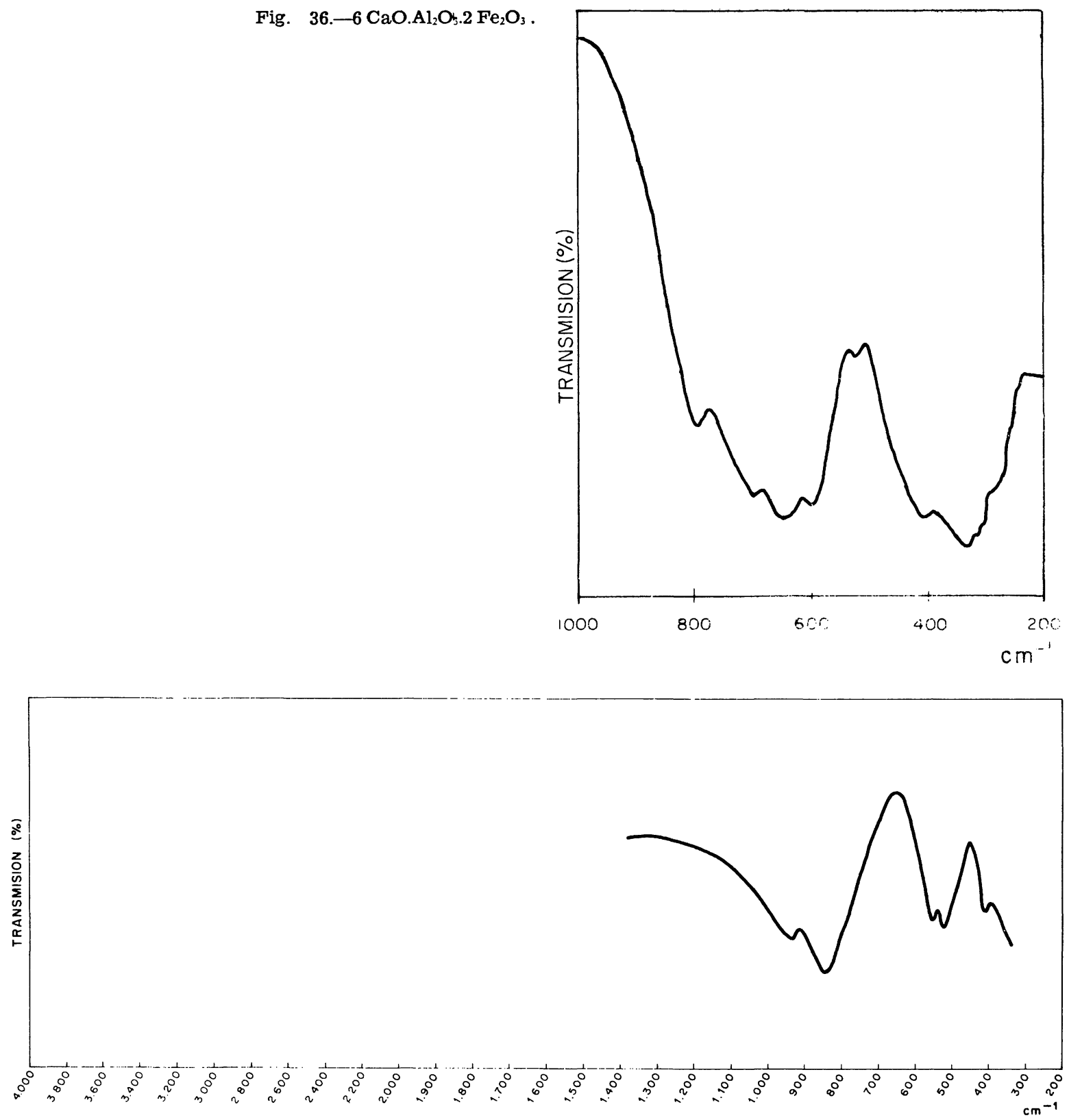

Fig. 37. $-2\left(2 \mathrm{CaO} . \mathrm{SiO}_{2}\right) \cdot \mathrm{CaF}_{2}$.

Fig. 38.--2 (2 $\left.\mathrm{CaO} . \mathrm{SiO}_{2}\right) \cdot \mathrm{CaSO} 4$.

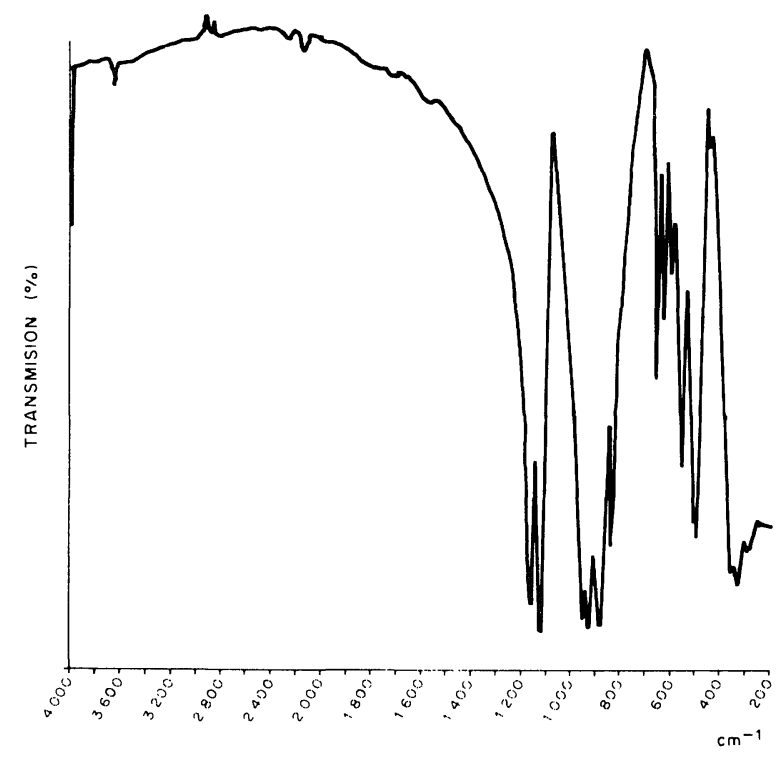




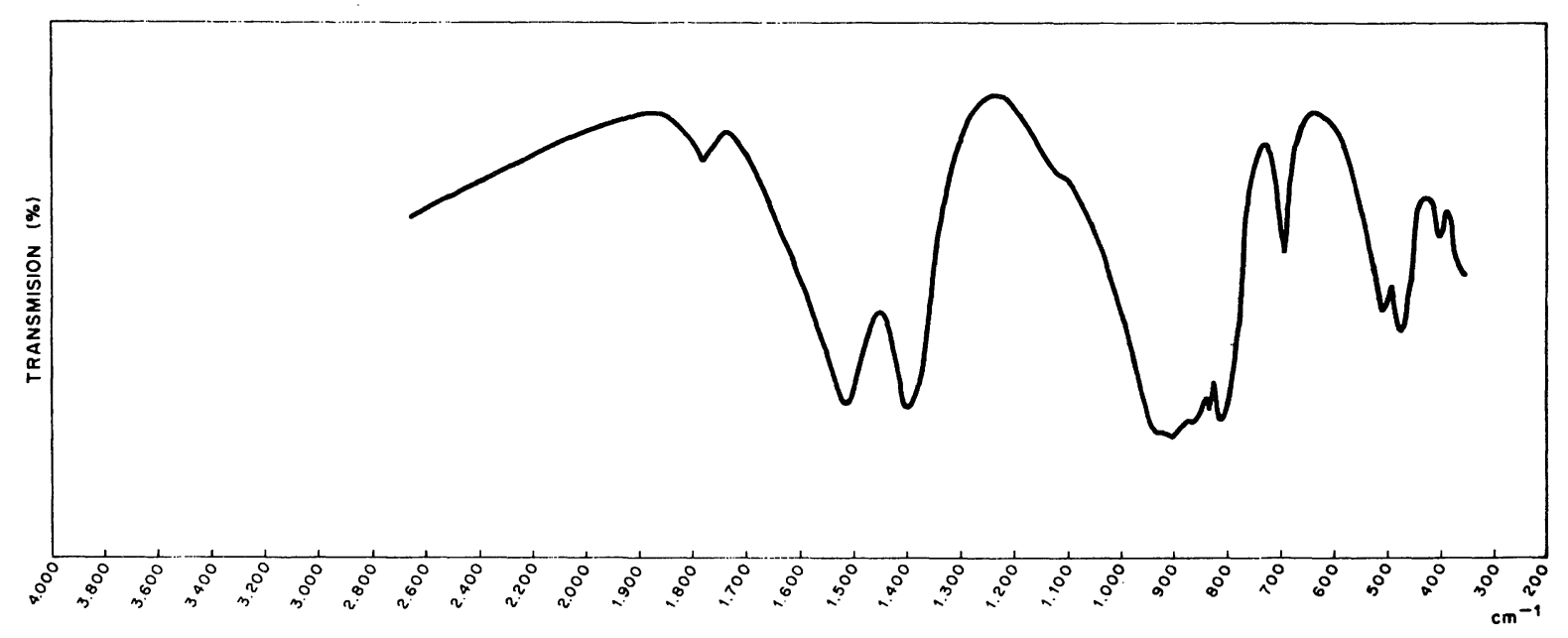

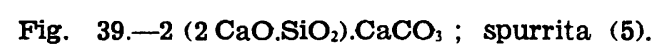

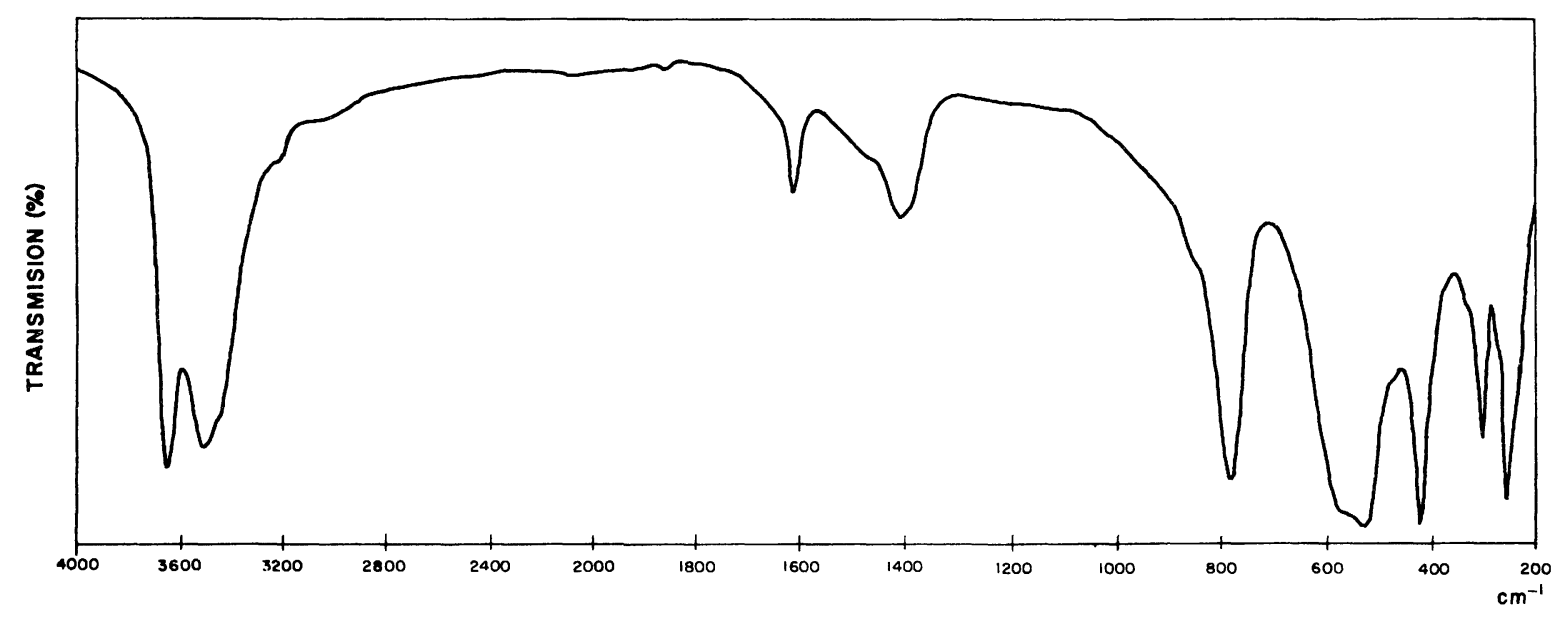

Fig. 40.-3 $\mathrm{CaO} \cdot \mathrm{Al}_{2} \mathrm{O}_{3} \cdot \mathrm{CaCl}_{2} \cdot 10 \mathrm{H}_{2} \mathrm{O}$; Sal de Friedel.

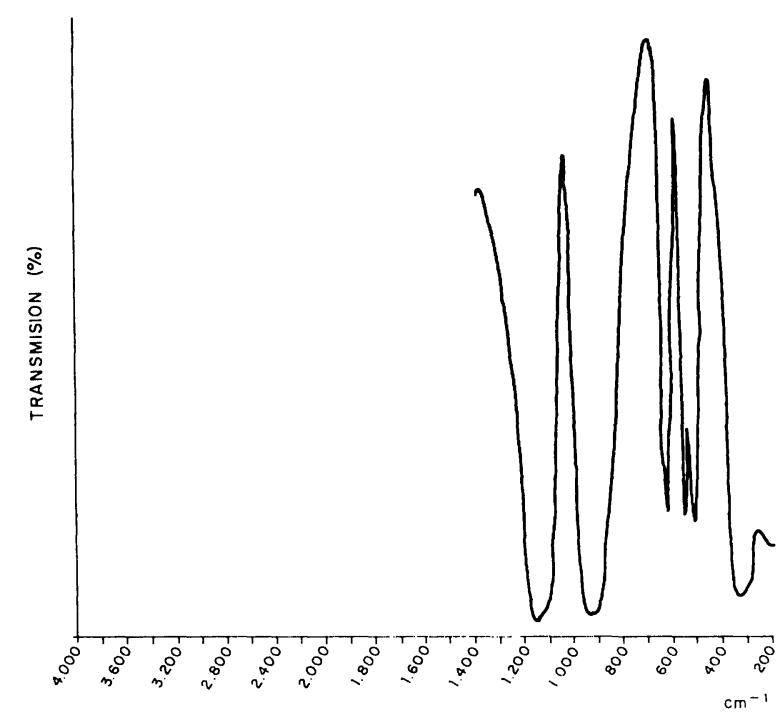

Fig. $41-3 \mathrm{Ca}_{2} \mathrm{SiO}_{4} \cdot 3 \mathrm{CaSO}_{4} \cdot \mathrm{CaF}_{2}$; fluorellestadita. 